\title{
On Interference Avoidance through Inter-Cell Interference Coordination (ICIC) based on OFDMA mobile systems
}

\author{
Chrysovalantis Kosta, Bernard Hunt, Atta UI Quddus and Rahim Tafazolli
}

\begin{abstract}
The widely accepted OFDMA air interface technology has recently been adopted in most mobile standards by the wireless industry. However, similar to other frequencytime multiplexed systems, their performance is limited by intercell interference. To address this performance degradation, interference mitigation can be employed to maximize the potential capacity of such interference-limited systems. This paper surveys key issues in mitigating interference and gives an overview of the recent developments of a promising mitigation technique, namely, interference avoidance through inter-cell interference coordination (ICIC). By using optimization theory, an ICIC problem is formulated in a multi-cell OFDMA-based system and some research directions in simplifying the problem and associated challenges are given. Furthermore, we present the main trends of interference avoidance techniques that can be incorporated in the main ICIC formulation. Although this paper focuses on 3GPP LTE/LTE-A mobile networks in the downlink, a similar framework can be applied for any typical multi-cellular environment based on OFDMA technology. Some promising future directions are identified and, finally, the state-of-the-art interference avoidance techniques are compared under LTEsystem parameters.
\end{abstract}

Index Terms-ICIC, Inter-cell resource allocation, Selective interference avoidance, Selective frequency/power reuse.

\section{INTRODUCTION}

The exponential growth in the number of communications devices over the past decade has set out new ambitious targets to meet the ever-increasing demand for user capacity in emerging wireless systems. However, the inherent impairments of communication channels in cellular systems pose constant challenges to meet the envisioned targets. In order to deal with the high cost and scarcity of suitable wireless spectrum, higher spectral reuse efficiency is required across the cells, inevitably leading to higher levels of interference.

The intra-cell (adjacent-channel) interference can effectively be mitigated in systems based on orthogonal frequency division multiplexing (OFDM). This intra-cell mitigation relies on appropriate physical layer design and good orthogonality of sub-carriers. On the other hand, there is considerable inter-cell (co-channel) interference from reusing the same frequency channels between neighboring cells. In such interference-limited systems, the cell-edge users are more susceptible to this inter-cell interference because, in addition to the high path loss, multiple strong interferences exist from nearby cells.
In the future, a mass deployment of overlaid networks, i.e. remote radio heads (relays) and low-power nodes (e.g. femtocells, picocells), is expected to enhance the radio coverage and considerably increase the quality of service (QoS) [1]. All of these supplementary networks, also known as heterogeneous networks (HetNets), jointly aim to extend the radio coverage in licensed bands and to support a large number of bandwidth-hungry multimedia services. However, their heterogeneity will cause higher inter-cell interference if their operation is not coordinated. Thus, investigation of intercell interference mitigation techniques is urgently required.

Both within the academia and the industry, the inter-cell interference can be treated by techniques of 1) randomization [2], 2) cancellation/rejection [3], 3) Coordinated Multi-Point transmission (CoMP) [4] 4) Multiple-Input Multiple-Output (MIMO) [5] and 5) Inter-Cell Interference Coordination/ avoidance (ICIC) [6]. In the first technique the interference is averaged across the whole spectrum via spreading sequences (e.g. scrambling, interleaving, or permutation codes), therefore it is not actually cancelled out but is spread across the system bandwidth. In contrast, the interference can be successfully rejected by using advanced signal processing based cancellation techniques. Even though these techniques are becoming popular, the complexity at the receiver side is still a challenge, particularly in the presence of multiple dominant interferers.

Using advanced multi-antenna techniques such as MIMO is another popular trend to increase the coverage and the overall system performance. Equally popular, the CoMP technique (similar to MIMO), which jointly processes and decodes the signals from multiple transmissions, is gaining high momentum in achieving significant performance gain and coverage optimization. However, the required processing and implementation complexity of these advanced processing techniques prevent them making a significant improvement to indoor coverage [7].

ICIC techniques, on the other hand, present a more feasible solution by applying restrictions to the radio resource management (RRM) block, improving favorable channel conditions across subsets of users that are severely impacted by the interference, and thus attaining high spectral efficiency. This coordinated resource management can be achieved through fixed, adaptive or real-time coordination with the help of additional inter-cell signaling in which the signaling rate 


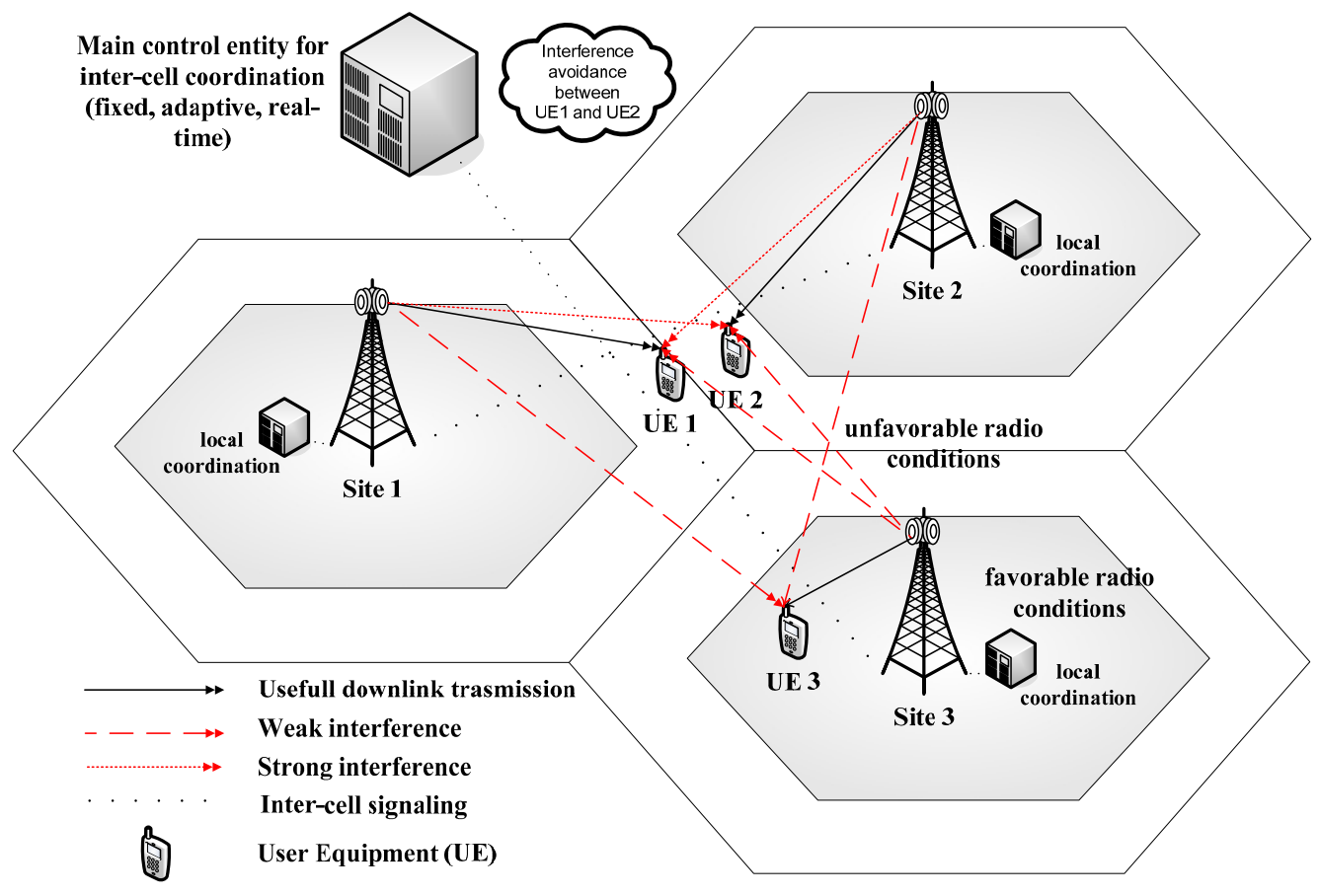

Fig. 1. Overview of the interference avoidance through inter-cell interference coordination (ICIC) problem in downlink macro cellular OFDMA systems

can vary accordingly. In general, inter-cell signaling refers to the communication interface among neighboring cells and the received measurement message reports from user equipments (UEs). Fig. 1 illustrates this concept using a small interconnected network. The figure also illustrates an interference avoidance example between UE1 and UE2.

Put simply, one-way to combat the interference experienced by critical users without any additional changes to the mobile standard is to employ a clustering reuse technique [8]. For instance, employing reuse- 3 technique can significantly reduce the interference; however, it will inevitably lead to a low spectral efficiency.

In this paper, our main focus is on transmitter side (downlink) ICIC techniques, and we provide a comprehensive overview and examination of the recent developments in these avoidance schemes. Compared with other surveys existing in the literature regarding interference mitigation and coordination [7] [9] [10] [11], we formulate a linear optimization problem for any typical multi-cellular environment based on OFDMA technology using binary/integer programming. Additionally, we provide a comprehensive performance comparison of the state-of-the-art in this area. Similar to these surveys, we give a clear understanding of the interference avoidance trends and a complete overview of employing these techniques using intercell coordination. To consolidate the knowledge generated in this area we provide a proper taxonomy of the investigated techniques. Furthermore, we underline some lessons learnt from the existing literature, highlight some important aspects of its evolution and suggest some future directions and challenges for further research. With the advent of HetNets, we believe that the employment of ICIC techniques becomes of paramount importance [7]. For a wider view on radio resource management (RRM), readers may also refer to these surveys \& tutorials [12] [14] [15] based on multi-user scheduling in OFDMA-based systems.

This paper is organized as follows: In Section II, we provide the general background context in multi-cellular networks. The binary multi-cell problem of using interference avoidance through inter-cell coordination in a multi-cell environment is formulated in Section III. In Section IV and Section V, we survey the major interference avoidance techniques together with further analysis of their employment using an ICIC framework. In Section VI, some ICIC techniques for emerging HetNets are discussed. In Section VII, we present the future evolution of ICIC techniques. A comparison of results of the state-of-the-art in interference avoidance is given in Section VIII. Finally, we conclude this paper with Section VIII.

\section{II.ESSENTIAL BACKGROUND}

In this section, first we review the key fading characteristics of the wireless channel of different communication terminals. Second, the widely used OFDMA radio interface in the downlink (DL) of LTE is analyzed along with its main features. Finally, the asymmetry that can be observed in 


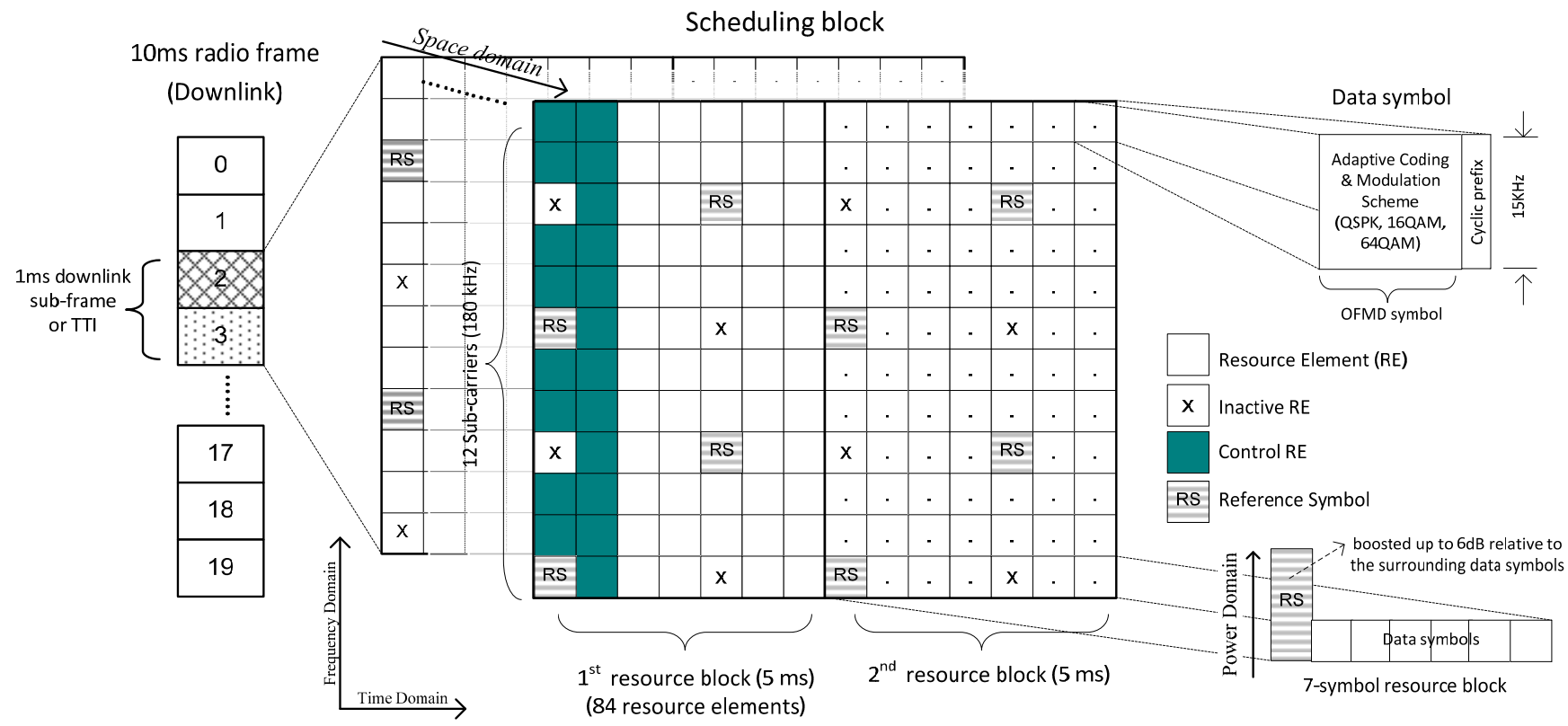

Fig. 2. The structure of a radio resource unit in downlink LTE-based systems (normal cyclic prefix \& with inactive/reserved resource elements in the case of a MIMO $2 \times 2$ configuration)

mitigating interference in downlink (DL) vs. uplink (UL) is explained.

\section{A. Wireless channel characteristics}

Generally, the wireless channel of different communication terminals can be expressed with a multiplicative propagation mechanism of three nearly independent fading components: path loss, shadowing and multipath. The fading effect of these can be scaled as 'large', 'medium' and 'small' and may have term as 'long, 'medium' and 'short', respectively [16]. For instance, the long-term and the large-scale fading effects of the wireless channel can be characterized by the path loss component. In a similar way, each of the remaining fading effects can be reflected respectively by the other two fading components.

Both the path loss and the shadowing component represent the 'position-dependent', and 'time-invariant' fading variations of the channel. By contrast, the multipath component is 'position independent' in which fading variations can be observed in all domains of the propagation channel i.e. time domain (TD), frequency domain (FD) and spatial domain (SD). This discrepancy of the above radio channel from mobile terminal to mobile terminal is mainly because of the multiple reflections of the same signal. As a result, multipath delay spread and frequency shift is experienced in each mobile terminal; thus, leading to rapid fluctuations within the properties of the received signal [17]. An extensive overview and analysis of modeling wireless communication channels is given in [18] [19].

In a multi-user scenario, we can then simply assume that the wireless environment varies statistically independently across all wireless terminals. With this statistical assumption, the likelihood of a deep wideband fade across all mobile terminals at a time is minimal. In other words, is almost certain that there is a sub-channel in the wireless channel of a user terminal with a non-deep fading sub-channel. Interestingly, the channel variability across different Resource Blocks (RBs) extends not only to the desired channel but also to the interference channel. Consequently, the exploitation of channel variations in all domains is highly favorable. Notably, the total system performance ${ }^{1}$ can also be improved by increasing the number of users [14]. Therefore, here we assume that the number of the users in all the investigated scenarios is fixed in order to moderate the changes in performance stemming from this channel diversity.

\section{B. Overview of LTE air-interface technology in the Downlink}

Next generation mobile systems such as 3GPP LTE, LTEAdvanced (LTE-A), and WiMax have adopted a radio interface based on OFDMA (Orthogonal Frequency Division Multiple Access) technology. However, a low peak-toaverage power ratio (PAPR) design based on SC-FDMA (Single-Carrier Frequency Division Multiple Access) technology is employed in UL LTE based systems. A major difference with earlier multiple access techniques, is that both OFDMA and SC-FDMA can possess dimensions of not only time and frequency but also space by means of multiple antennas (e.g. MIMO). However, an efficient linear amplifier

\footnotetext{
${ }^{1}$ Here, the system performance denotes the total cell throughput. Although the system performance may be improved by increasing the number of users due to channel diversity, the achievable user throughput is decreased.
} 
for the RF transmitter is certainly a main issue for research, since the candidate scheme (OFDMA) exhibits high PAPR [20].

A detailed resource structure of the radio frame for DL LTE deployments is shown in Fig. 2 [21]. The largest radio resource unit in DL is a radio frame consisting of 10 subframes (or blocks), where each one is $1 \mathrm{~ms}$ long in duration. A sub-frame (or slot) is considered the minimum allocable element in scheduling, also commonly known as a transmission time interval (TTI). This scheduling block can be seen as two consecutive resource blocks (RBs), which each $\mathrm{RB}$ is sub-divided (in the frequency domain) into 12 subcarriers keeping $15 \mathrm{kHz}$ sub-carrier spacing (thus occupying a total of $180 \mathrm{kHz}$ ). In the time domain, the RB can be divided into 6 or 7 OFDM symbols depending on which extension of cyclic prefix (normal or extended) is chosen. This extension mainly serves as guard interval between two consecutive symbols and helps to eliminate the residual intersymbol interference (ISI) caused by multi-path propagation. In the case when normal cyclic prefix is employed, a grid of 84 resource elements (REs) is formed per RB. To convey multiplexed data reliably, adaptive modulation and coding (AMC) may be used in each RE i.e. QPSK 16-QAM or 64QAM along with a coding rate. High AMC such as 256 QAM or even higher is envisaged to benefit supplementary networks with improved indoor coverage. However, further investigation is needed to clarify the level of improvement and technical problems involved in adopting a high AMC.

Cell-specific reference symbols (RS) may be multiplexed in the case of the pilot channel for OFDMA-based systems in order to facilitate the channel estimation process between transmitter and receiver [22]. Here, as depicted in Fig. 2, a pattern close to 'diamond' can be shaped to benefit this process in varying mobile environments [23]. To diminish the co-antenna interference in DL, alternative REs are currently inactive (also known as inactive RE). In reference signal structure, the FD positions of RS should also vary among neighboring cells, irrespective of type, to avoid any conflicts. Additionally, to track more accurately both the serving and the interfering cell-specific sequences, it can be boosted up to $6 \mathrm{~dB}$ compared with surrounding data symbols [24]. The orthogonality of the cell-specific reference sequences can be viewed as a key component for the performance of dynamic ICIC techniques. Therefore, the design of optimal pilot sequences is one of the more important issues that operators must deal with before small-cell networks become widely deployed [25].

A constant power allocation policy is considered over all RBs in order to avoid any additional discrepancy in the radio channel among different mobile terminals. The maximization of the spectral efficiency in a multi-cell OFDMA-based scenario requires a water-filling algorithm, where the exact solution can have prohibitive computational complexity. However, a constant-power energy distribution for OFDMA- based deployments can have a negligible performance loss compared with the water-filling algorithm [26]. Furthermore, a recent study has shown that in dense mobile systems such as femtocells a fixed power allocation policy can perform better than conventional schemes [27]. Therefore, the power allocation is considered fixed over the time in most situations based on DL LTE system.

\section{Interference Mitigation through interference avoidance (Data link regaining through interference mitigation)}

In general, interference avoidance refers to the use of power-frequency, or even time domain restrictions in order to minimize the dominant interferers to a subset of disadvantaged users. In order to provide a suitable coordination framework to the network provider, the distribution of the radio resource restrictions can be classified mainly as centralized, semicentralized and decentralized. Therefore, interference avoidance is considered a promising interference mitigation technique without introducing major changes on the design of the air interface of already existing standards [10].

As discussed earlier, a straightforward approach to reduce inter-cell interference is through conventional frequency reuse- $\mathfrak{R}$ systems [30]. By allocating $\mathscr{R}$ non-overlapping frequency groups in each cell, the inter-cell interference is avoided by a certain ratio compared with universal frequency reuse system (reuse-1). However, the ratio only increases logarithmically as the reuse factor $(\mathfrak{R})$ is increased. Another drawback of the reuse- $\Re$ system is that it always causes a steep degradation in the overall system performance, since the available radio resources in each cell is diminished by the reuse factor [31]. One way to improve the critical throughput without jeopardizing the entire cell performance is to use a selective interference avoidance technique [32]. Selective interference avoidance is a bandwidth-efficient approach of using individual reuse techniques in different cell areas or even in different user groups. More details and techniques for selective interference avoidance are given in the next section.

\section{Heterogeneity of Interference in DL vs. UL}

Notable differences can be observed when inter-cell interference in the downlink (DL) is compared to the uplink (UL). Generally, due to the channel variability across the RBs and traffic demand, the interference is more evenly propagated in both spatial and time domains in DL compared to UL. In the DL case, the inter-cell interference experienced by a stationary user terminal in a cell is constant over short periods of time, given that the base station transmits across most of the given spectrum to the resolution of a sub-carrier with a relatively high transmit power [33]. However, this is not the case in the UL since a mobile terminal can have an adaptive power profile in different locations and different users may transmit in different (contiguous) parts of the bandwidth [34].

In any case, DL or UL, it is expected that a traffic-load 
adaptive ICIC technique will have a larger performance gain [35]. However, it is important to note that there are more opportunities for a coordination gain in the UL irrespective of selected avoidance technique [25]. In this paper, most of the avoidance techniques apply equally well in UL without significant modification unless otherwise mentioned.

\section{General Problem Formulation of ICIC In Multi- CELL OFDMA-BASED SYSTEMS}

By the geometric nature of a typical cellular system, celledge UEs are the most disadvantaged members of the network, as in addition to the higher path loss experienced within the attached sector, significant interference is received from nearby cells. Any optimal or suboptimal RRM with an objective function to maximize user throughput in a networkwide range will avoid these disadvantaged users, since their part to the overall throughput is minimal [39]. Therefore, by employing selective interference avoidance on such users, the network can effectively extend their minimum data rates.

We consider a LTE system based on OFDMA, a set of inter-connected mobile network with total $S$ eNodeBs (eNBs), $K$ UEs and $N$ RBs. Let us assume the set of eNBs as $\mathbb{S}=\{1,2$, $\ldots, S\}$. It has been assumed that the transmit power on each $\mathrm{RB}$ is the same and fixed. However, specific RBs termed as 'restricted' are scheduled with a limited power or are not scheduled at all. To define the set of the possibilities of ingroup interfering eNBs we use the powerset expression $P()$. For example, the powerset of $\{A, B\}$ is: \{\}$,\{A\},\{B\},\{A, B\}$. Note that the empty set \{\} denotes that no interference mitigation scenario is considered. Following this, we can construct all possible subsets of eNBs that can interfere with eNB $i$ using the following notation $P(\mathbb{S} \backslash\{i\})$. Note that the powerset $P(\mathbb{S} \backslash\{i\})$ has $|P(\mathbb{S} \backslash\{i\})|=2^{S-1}$ combinations.

In order to reduce the large number of possible interfering scenarios it has been also assumed that the system uses cellspecific orthogonal reference sequences. Therefore, the UEs can measure the separate levels for different dominant sources of interference. With the help of an index $g^{i}$, we denote the subsets of the powerset of dominant interfering eNBs. Furthermore, with the variable $h^{i}$ we denote the list of eNBs that they may have a dominant interfering scenario with the eNB $i$. In order to capture the channel diversity across the interference channel, the symbols $g^{i, n}$ and $h^{i, n}$ may be used, respectively. Then the SINR is conveniently constructed with a list of dominant $\left(j \in g^{i}\right)$ and non-dominant $\left(j \notin g^{i}\right)$ interferences as follows:

$$
\gamma_{k, n}^{i, g^{i}}=\frac{P_{a / b} \cdot H_{k, n}^{i}}{P_{a} \cdot \sum_{\substack{j \neq i \\ j \neq g^{i}}} H_{k, n}^{j}+P_{b} \cdot \sum_{\substack{j \neq i \\ j \in g^{i}}} H_{k, n}^{j}+N_{w}}
$$

Here, $\gamma_{k, n}^{i, g^{i}}$ denotes the instantaneous SINR at UE $k$ that is connected to eNB $i$ excluding the dominant interfering eNBs which belong in the interfering scenario group $g^{i}$. $H_{k, n}$ denotes the channel gain which includes all key fading components (path loss, shadowing, and multipath) that UE $k$ experiences on RB $n$. We use super-indexes $i$ and $j$ to represent the desired and interfering link, respectively. For simplicity in this formulation, we consider that the eNBs in the dominant interfering eNB scenario group $g^{i}$ can either transmit with a predefined limited power or not transmit at all. Therefore, the parameter $P_{a}$ denotes the unrestricted transmit power while $P_{b}$ denotes the restricted transmit power. With the notation $P_{a / b}$, we stress that normal or limited power can be allocated to the scheduled RB. In the case where the restricted RB is not scheduled at all $\left(P_{b}=0\right)$, normal power should be allocated $\left(P_{a / b}=P_{a}\right)$ on the non-restricted RBs. For convenience, a list of symbols used in this paper is provided in Table 1.

We define $f($ ) as a mapping function where the instantaneous SINR is converted through achievable data rate as:

$$
r_{k, n}^{i / g_{i}}=f\left(\gamma_{k, n}^{i / g_{i}}\right)
$$

The binary problem of interference avoidance through ICIC in an interference-limited multi-cell system is formulated below:

$$
\begin{array}{ll}
\operatorname{maximize} \quad & \sum_{i} \sum_{g^{i}}\left(\sum_{k=1}^{K} \sum_{n=1}^{N} U_{k, n}^{i, g^{i}} \cdot \rho_{k, n}^{i, g^{i}}\right) ; \\
& U_{k, n}^{i, g^{i}}=\left(r_{k, n}^{i, g^{i}}\right)^{x} \cdot\left(d_{k}^{i}\right)^{y} ; \quad x, \mathrm{y} \in \mathrm{N}^{+} \\
\text {subject to } & \underbrace{\sum_{g^{i}} \sum_{k=1}^{K} \overbrace{\rho_{k, n}^{i, g^{i}}}^{V 1}}_{X 1}+\underbrace{\sum_{j \in h^{i}} \sum_{g^{j}} \sum_{i} \sum_{k=1}^{K} \frac{\rho_{k, n}^{j, g^{j}}}{\left|h^{i}\right|}}_{X 2} \in\{0,1\} ; \forall n \\
& X 1, V 1, X 2, V 2 \quad \in\{0,1\} .
\end{array}
$$

The optimization problem in (3) can be used with the multioption utility measure in order to favor a varying degree of emphasis on user throughput and fairness, and therefore $x$ and $y$ exponents are defined, respectively. For instance, the $\mathrm{x}=1$ and $y=0$ option or other non-zero $x$ options, aim to maximize the aggregate sector throughput as the utility shows minimal benefit to the deprived users. Therefore, other non-zero $y$ options of the utility can give more gain to these users. Here, we define the demand factor as the average throughput across all UEs divided with the average throughput of UE $k$.

The terms $X 1 \& X 2$ in constraint (4) are binary-integer type and jointly indicate that the complex ICIC problem belongs in binary/integer programming. The variable $V 1$ implies by 1 or 0 whether the RB $n$ is assigned for UE $k$ on eNB $i$ excluding the dominant interfering eNBs $\in g^{i}$ or not. The term $X 1$ ensures that each RB can be assigned to one user for each dominant interfering eNB scenario group $g^{i}$, which is restricted. On the other hand, the term $X 2$ expand this interrelationship for all eNBs $j \in h^{i}$ which they have an interfering scenario with eNB $i\left(g^{j} \ni i\right)$. The denominator $\left|h^{i}\right|$ under the variable $V 2$ normalizes the term $X 2$ to 1 . The significance of 
the denominator $\left|h^{i}\right|$ is to allow more than one candidate (eNB $j$ ) to transmit in the case when the eNB $i(X 1=0)$ does not transmit. Note that each eNB (interfering or noninterfering) is allowed to transmit only to one user and one interfering scenario group. Therefore, in order to maximize the total utility the objective function is accumulated over all the variables.

In few words, both $X 1 \& X 2$ indicate that if the RB $n$ is not restricted, it can therefore be allocated to one UE or if the RB $n$ is restricted, it can then benefit more than one candidate. However, in order to understand the inter-relationship of an interfering eNB with other eNBs let us assume a simple scenario where the eNB $C$ is the interfering eNB of both eNB $A$ and eNB $B$ and the eNB $A$ is the interfering eNB of eNB C. Therefore, we consider that $g^{A} \rightarrow\{\{\},\{C\}\}, g^{B} \rightarrow\{\{\},\{C\}\}$, $g^{C} \rightarrow\{\{\},\{A\}\}, h^{A}=\{\{C\}\}, h^{B}=\varnothing$ and $h^{C}=\{\{A, B\}\}$. Then, the problem is formulated as:

$$
\begin{array}{ll}
\text { maximize } & \sum_{i}^{\{A, B, C\}} \sum_{g^{i}}\left(U^{i, g^{i}} \rho^{i, g^{i}}\right) ; \\
\text { subject to } & \sum_{g^{C}}^{\{\{,\{A\}\}} \rho^{C, g^{C}}+\sum_{j \in h^{i}}^{\{A, B\}} \sum_{\left.g^{j} \ni C\right\}} \frac{\rho^{j,\{C\}}}{2} \in\{0,1\} ; \\
& \sum_{g^{A}}^{\{,\{C\}\}} \rho^{A, g^{A}}+\sum_{C \in h^{A}}^{\{C\}} \sum_{g^{C} \ni A}^{\{\{,\}\}} \rho^{C, g^{C}} \in\{0,1\} ; \\
& \rho^{A, g^{A}}, \rho^{B, g^{B}}, \rho^{C, g^{C}} \in\{0,1\} .
\end{array}
$$

For simplicity, the notations $k$ and $n$ are omitted in this example. The constraint in (6) may be expanded as follows:

$$
\begin{aligned}
& \rho^{C,\{\}}+\rho^{C,\{A\}}+\frac{\rho^{A,\{C\}}}{2}+\frac{\rho^{B,\{C\}}}{2} \quad \in\{0,1\} ; \\
& \rho^{A,\{\}}+\rho^{A,\{C\}}+\rho^{C,\{A\}} \in\{0,1\}, \\
& \text { or } \\
& \rho^{A,\{}, \rho^{B,\{\}}, \rho^{C,\}} \in\{0,1\} .
\end{aligned}
$$

The condition in (8) denotes the terms of condition by employing interference avoidance at eNB C. In a similar way, the condition in (9) denotes the terms of condition by employing interference avoidance at eNB A. Clearly, either eNB C or eNB A \& eNB $B$ may have additional date rate gain by employing interference avoidance. One scenario is when we restrict eNB $B$ in order to apply interference avoidance to both eNB $A$ and eNB $B$. Note that eNB $A$ and eNB $B$ are interfering with each other but not in a dominant way so the utility of the network with both eNB $A$ and eNB $B$ transmitting is higher. However, all eNBs may transmit in the case where no interference mitigation scenario is assumed \{\} , i.e. the constraint in (9).

The formulation can be extended in order to consider a number of other issues. For example, with constraint (10) the number of RBs each UE is assigned is limited by $\mathbb{N}$. Moreover, to satisfy user minimum data rate we use constraint (11) and to apply power constraints we use constraint (12).

\section{TABLE 1: List OF SyMBOLS}

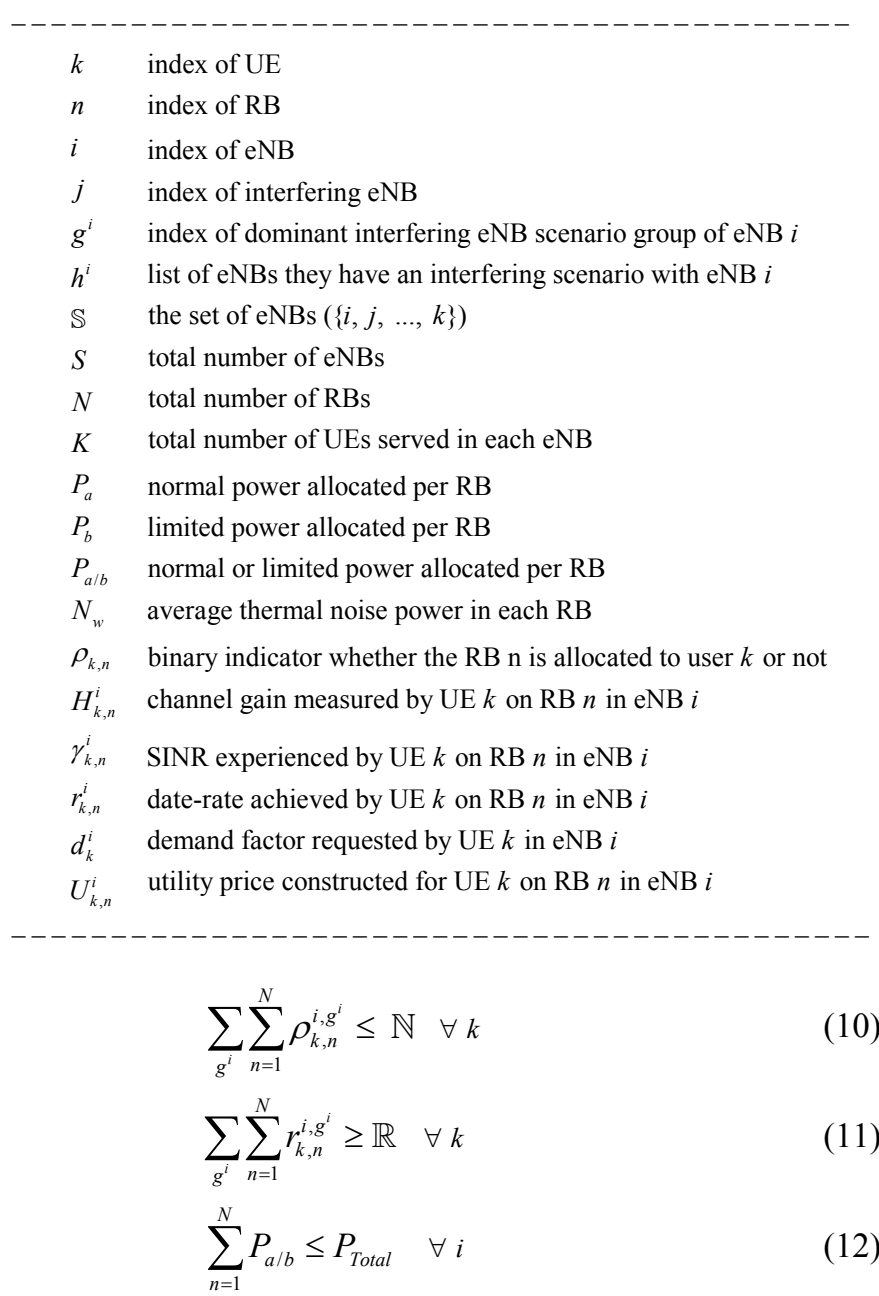

The complexity cost quoted by the above binary problem increases significantly with the number of $i, k, n$ variables and number of subsets in $g^{i}$ and $h^{i}$. Solving this binary combinatorial optimization problem on a network-wide scale and accounting for all variables can result in a large computational burden. Therefore, it is very important to solve the ICIC problem as efficiently as possible.

One main challenge associated with problems belonging to the class of binary linear programming (LP) is that they require an extensive search path. Prolonged extensive search of a linear problem can be reduced by introducing a number of tighter constraints or cuts [36] [37] [38]. However, generating cuts can be a dubious step as it may increase the size of the original problem without removing significant search paths. Nevertheless, effective binary/integer-based optimization tools can approximate the solution quickly for small and practical scenarios by employing branch-and-cut algorithms [40] [41].

In order to reduce the complexity related to the scalability of the problem we can introduce a number of additional assumptions, which may have some performance cost to the original problem. Binary LP problems with limited search path can be relaxed [42] and solved in polynomial time. LP relaxation is the problem that arises when binary variables are 


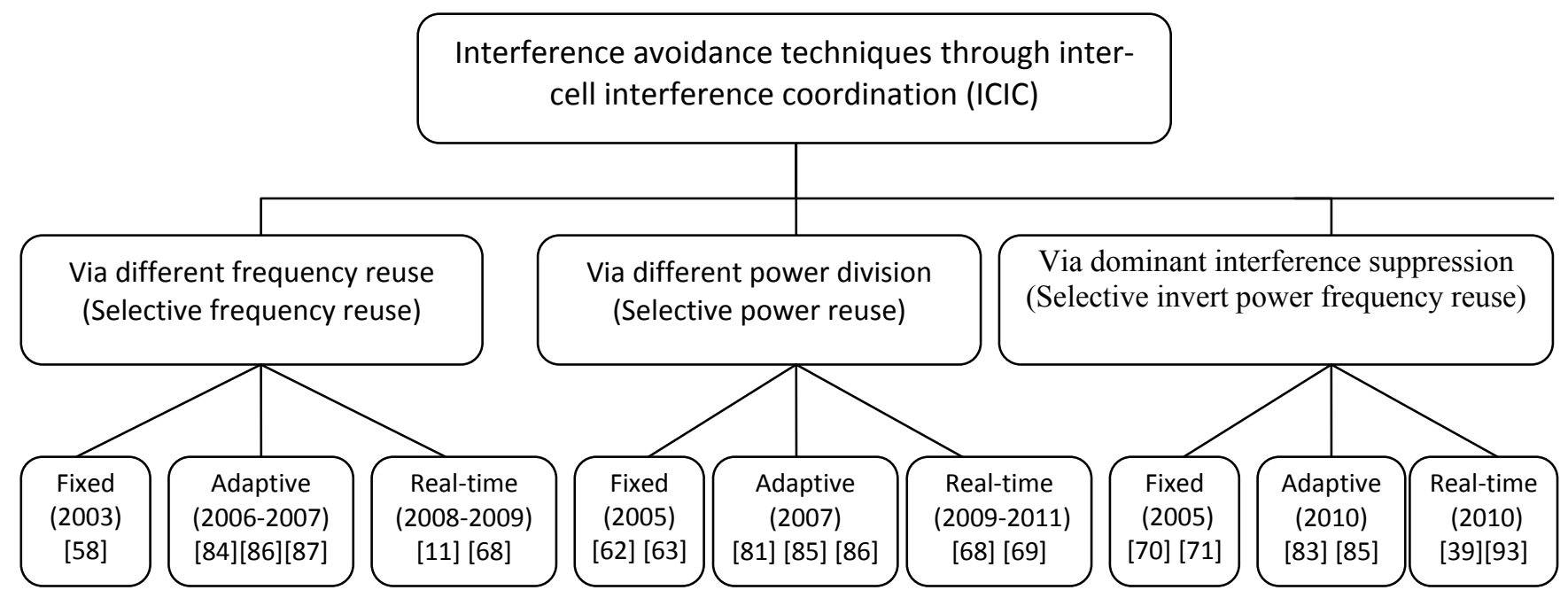

Fig. 3a. Main developments of selective interference avoidance for macro OFDMA systems

replaced with real variables belonging to interval [0 1$]$. For instance, the constraint in (4) can be simplified by removing the denominator $\left|h^{i}\right|$ and relaxed as,

$$
\begin{array}{ll}
\text { subject to } & \sum_{g^{i}} \sum_{k=1}^{K} \rho_{k, n}^{i, g^{i}}+\sum_{j \in h^{i}} \sum_{g^{j} \ni i} \sum_{k=1}^{K} \rho_{k, n}^{j, g^{j}} \in[0,1] ; \forall n \\
& \rho_{k, n}^{i, g^{i}}, \rho_{k, n}^{j, g^{j}} \quad \in[0,1] .
\end{array}
$$

Now the constraint (9) of the reformulated LP problem is a special case, i.e. problem with totally unimodular matrix specifications [43], where its relaxed solution is also the optimal solution to the initial problem. This means that there is a small integrality ${ }^{2}$ gap between the reformulated binary problem and of its relaxation. In this case, one optimality issue of substituting the constraint (9) with the relaxed constraint (4) is that the reformulated problem does not benefit more than one of the eNB candidates that are requesting restrictions. For example, assume the previous scenario where both candidates, i.e. eNB $A$ and eNB $B$, will be benefited if eNB $C$ does not transmit on a particular RB. In the case that the eNB $C$ is restricted to this $\mathrm{RB}$, inevitably only one candidate is the beneficiary of the above assumption. In reality by adopting such assumption, there is a low performance cost to the original problem since it is considered sufficient by accounting for only the strongest candidate. Moreover, the performance degradation can be further reduced by executing afterwards a routine to search for and enable all the disadvantaged eNBs that have been neglected.

A number of recent studies [44] [45] emphasize that the ICIC problem can be converted and solved as a minimum cost network flow (MCNF) optimization problem. The motivation of converting such a problem to a MCNF stems from the necessity in solving the ICIC problem faster than a generic LP

\footnotetext{
${ }^{2}$ Integrality gap is defined as the maximum ratio between the solution quality of the binary/integer problem and of its relaxation.
}

solver. However, the disadvantage of this transformation is that the current algorithms available in the literature limit the ICIC solution only up to the $1^{\text {st }}$ dominant interference. This implication is significant for high-level interference scenarios such as femtocells where the mitigation of the $2^{\text {nd }}$ and $3^{\text {rd }}$ dominant interference becomes essential.

Another way to reduce the computational complexity of above ICIC formulation is to break it down into several subproblems at a time with a small set of interfering eNBs and allocated RBs [39]. Furthermore, the ICIC problem can be seen as a combined problem of two smaller sub-problems i.e. the intra-cell RRM as the local problem and the inter-cell resource restriction as the network problem [46]. To benefit scalability, the network problem can be extended to single master and multiple slave sub-problems by employing primal/dual decomposition from optimization theory [120]. One issue associated with the optimality of primal/dual decomposition is that it requires the problem to be not only relaxed but also in a convex form. However, we may improve the convexity by transforming some functions into convex form. For example, let us redefine the user operating rate function in (2) by assuming a log-transformed rate [13] which is not operator dependant as,

$$
R=B \log _{2}\left(1+\frac{\operatorname{SINR}}{L}\right) \quad L=-\frac{1.5}{\operatorname{In}(5 \cdot \mathrm{BER})} .
$$

Here, $R$ is the maximum amount of data that can be conveyed in terms of SINR, B is the allocated bandwidth and $L$ is the SNR gap to data-rate, which is linked via a particular target bit error rate (BER) [14]. The rate region $\mathrm{R}$ is now convex.

The ICIC formulation provided in this section gives an optimal resource allocation by employing the concept of interference avoidance. It enables us to capture the interrelationship of all eNBs in a specific user scenario. By using the general ICIC formulation as an optimization problem, any static ICIC schemes can be dynamically extended at cost of 
Interference avoidance techniques through intercell interference coordination (ICIC)

Via self-organizing channel reuse (Self-organizing frequency reuse)

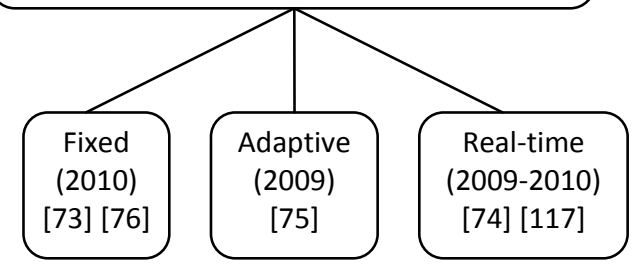

Fig. 3b. Main developments of selective interference avoidance for femto OFDMA systems

inter-cell signaling. Furthermore, we note that optimal ICIC will maintain an important pathway in the literature since it presents a critical benchmark for evaluating the performance of novel low-complexity centralized and semi-centralized algorithms.

It is important to mention that without significant modifications, the ICIC formulation is applicable to cells with different cell-size and even to indoor/outdoor femtocells. In the case of femtocells, a number of additional extensions may be applied in order to deal with a number of issues (ref. section VI). For instance, in order to minimize the number of restrictions to the main network, a supplementary eNB may not be permitted to request resource restrictions to a main eNB, whereas the opposite may not be true.

\section{OVERVIEW of Selective INTERFERENCE AVOIDANCE TECHNIQUES FOR OFDMA-BASED SYSTEMS}

Selective interference avoidance has received a lot of attention not only from the scientific community but also from standardization institutions and organizations [9]. In general, the research problem of interference avoidance pertains to the allocation of non-restricted radio resources to the cells for the serving users. As described earlier, the radio resource in a cellular network is allocated in terms of frequency, timespace, and power domains. However, the general focus of this research study is to look at how selective interference avoidance through inter-cell frequency and power arrangements can be beneficial primarily to critical members of the network and secondarily to the overall system. For the readers interested for further study, some early attempts in the area of inter-cell coordination may be found in [47] [48].

In order to separate and illustrate the concept of selective interference avoidance we use a simple cellular model as a miniature of a greater mobile network. In conjunction with this model, a group-specific power profile is defined over the whole bandwidth of which each eNB group can transmit at each frequency group over time. Subsequently the RBs from each frequency group can be selectively assigned, either to mobile terminals which are camped to a certain geographic area or to a set of terminals that are members of a bigger mobile group. Different criteria can be exercised in ranking one mobile terminal over another, or in identifying the lowcoverage areas. The three most popular ranking criteria are as follows: distance-based, utility-based, geometry-based (i.e. the ratio of pilot signal between the desired and the interfering sectors) [49]. Here, we consider this critical area as a sub-cell region that may not be physically bound to a certain geographical area and can consist of multiple disjoint sub-cell regions. Furthermore, to avoid any under-utilization of radio resources or to benefit multi-user diversity, the barrier to borrowing RBs from other frequency groups among different sub-cell regions can be lifted [50]. In other words, if some members of a sub-cell region opportunistically find some radio resources of another frequency group highly favorable they can utilize these, irrespective of their initial consideration.

Fig. 3 shows the main classes of interference avoidance techniques developed for macro OFDMA-based systems, further details of which are given in the next sub-section. The graphical model for these techniques is depicted in Fig. 4 \& Fig. 5. Note that all interference avoidance techniques are applicable in the context of ICIC. However for simplicity of illustration, we display the avoidance techniques in their fixed form (i.e. having fixed policy of how frequency groups are set) and consistent with tri-sectorized macro cellular networks. Additionally, in order to observe the utilization of radio spectrum we use the effective frequency reuse (eff. reuse) measure, which shows the amount of spectrum reused across the given geographical network area. For fair comparison, the total transmit power per sector is kept fixed and the same for all schemes.

Theoretically, there is no limitation on the number of concentric tiers (or rings) into which a cell area is split. It was shown in [51] that avoidance techniques can asymptotically double the system capacity as the number of concentric rings of a cell increases to infinity. In a similar way, many authors in [52] [53] [54] define up to three non-overlapping groups of users corresponding to three concentric tiers in order to provide improved inter-cell interference mitigation. However, our main considerations using selective avoidance through reuse techniques are limited to two sub-cell regions, namely, cell-edge and cell-center in order to categorize the given QoS to deprived and non-deprived users, respectively. The main trends and performance considerations are summarized in Table 2.

\section{A. Interference avoidance through different reuse factor (Selective frequency reuse)}

This form of selective interference avoidance was initially 
Selective frequency reuse

$$
P_{\text {high }}=3 \cdot P
$$
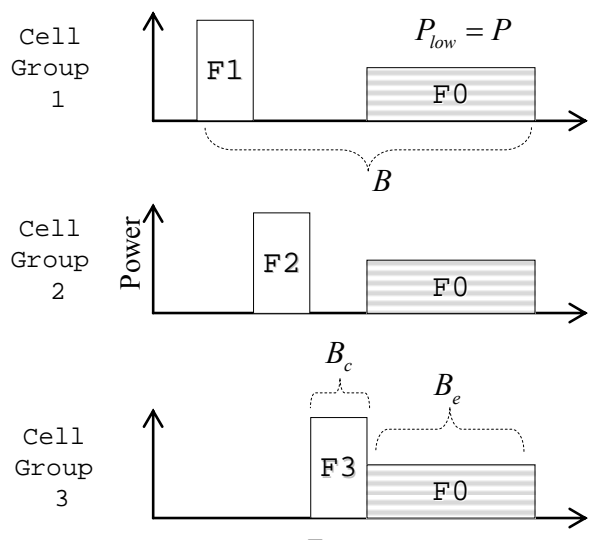

Frequency

or $P_{\text {high }}=P_{\text {low }}=P \times E f f . F R$

$$
\text { Eff. FR }=\frac{B}{B_{c}+B_{e}}
$$

Example of selective frequency/power reuse in a

tri-sectorized site.

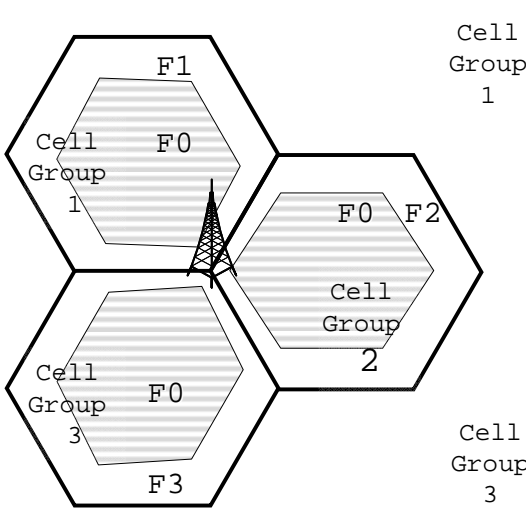

3

$1<$ Eff.FR $<3$
Selective power reuse

$$
P_{\text {high }}=a \cdot P
$$
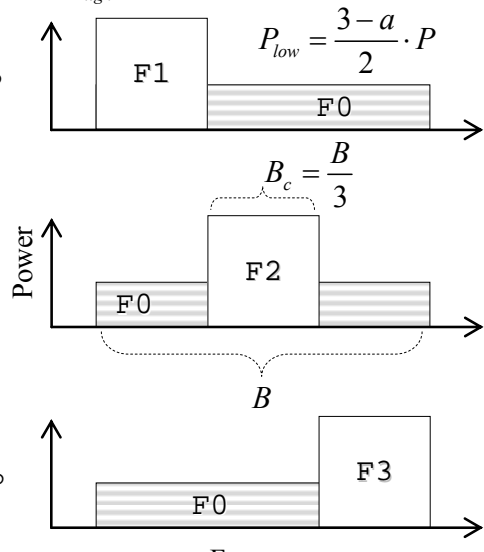

Frequency

Eff.FR $=\frac{P_{\text {high }}}{P_{\text {low }}}=\frac{3-a}{2 a} \quad 1<a<3$

Figure 4. Example of selective frequency (left) and power (right) reuse technique in a tri-sectorized site. Both selective interference avoidance techniques aim to minimize the inter-cell resource collisions to three major deprived regions (depicted as F1, F2 and F3) in relation to other cell groups across the network. In order to control the amount of radio resources in the deprived region, eff. reuse (effective reuse) measure is calculated in each case and its value can vary from one (reuse-1) to three (reuse-3).

Invert selective frequency/power reuse
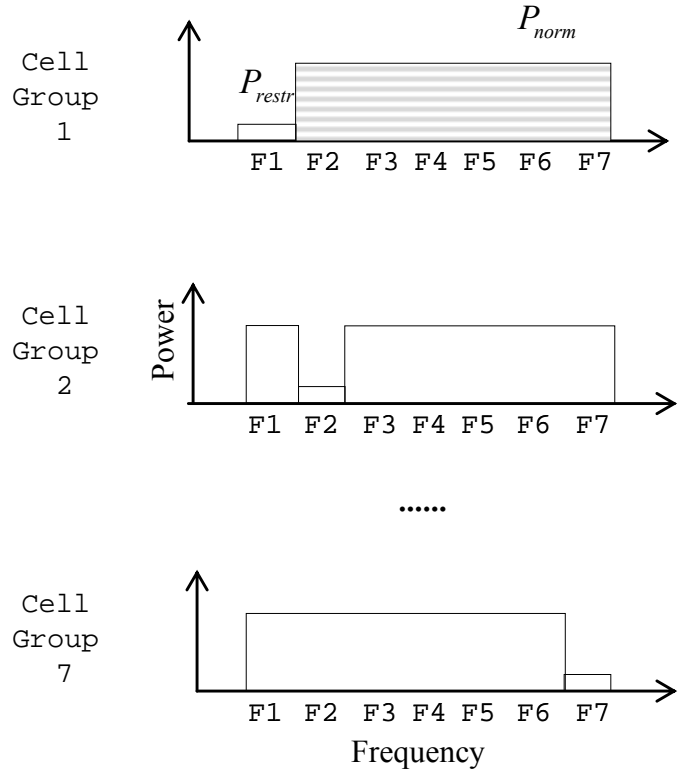

$$
P_{\text {restr }}=\beta \cdot P \quad P_{\text {norm }}=\frac{7-\beta \cdot P}{6} \quad \text { Eff } . F R=\frac{7-\beta}{6}
$$

Example of this technique in tri-sectorized sites

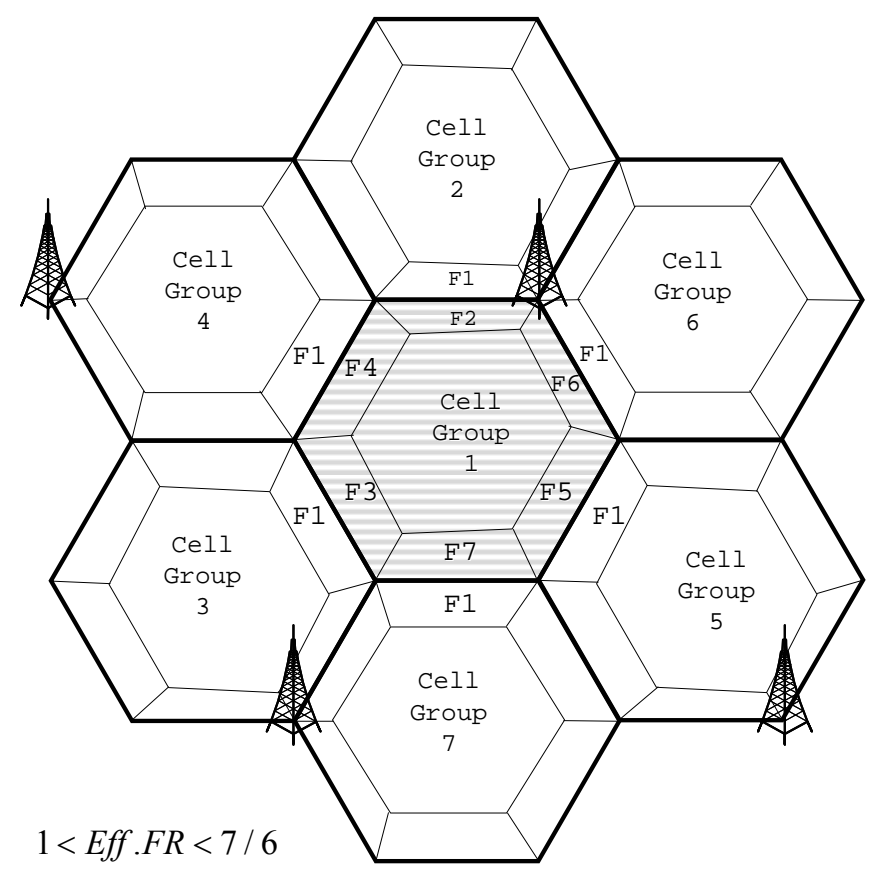

Figure 5. Example of invert selective frequency/power reuse technique in tri-sectorized cell networks. In order to increase the channel quality in all frequency groups, $F X, X \in\{1, \ldots, 7\}$, all surrounding sectors do not transmit full power to 1 of the 7 frequency groups in turn. Therefore, the cell-edge users of cell group 1 can be assigned to the frequency group to which transmit power is restricted by the nearby neighbor. The value of eff. reuse measure can vary from one (reuse-1) to 7/6 (reuse-7/6).

introduced in 1983 by Halpern in [55] for circuit switched networks; since then it has been widely used in Global System for Mobile communication (GSM) networks [56] [57] and in OFDMA-based systems [58]. Note that within the standardization activities of various forums (e.g. 3GPP LTE, WiMax Forum), the static form of this reuse technique is referred as fractional frequency reuse (FFR). The rationale behind interference avoidance through different frequency 
TABLE 2. TRENDS AND CONSIDERATIONS OF SELECTIVE INTERFERENCE AVOIDANCE

\begin{tabular}{|c|c|}
\hline TECHNIQUE & GENERAL CHARACTERISTICS AND PERFORMANCE CONSIDERATIONS \\
\hline $\begin{array}{l}\text { Selective } \\
\text { frequency } \\
\text { reuse }\end{array}$ & $\begin{array}{l}\text { A mixtures of two different frequency reuses, usually reuse- } 3 \text { and reuse-1, is employed for deprived and } \\
\text { non-deprived users, respectively. Compared with selective power reuse, a higher gain can be seen for the } \\
\text { deprived users in reuse- } 3 \text { environment and the gain can be enhanced further through a channel aware } \\
\text { scheme. However, it may suffer from under-utilization of radio resource due to the higher reuse measure. }\end{array}$ \\
\hline $\begin{array}{l}\text { Selective } \\
\text { power } \\
\text { reuse }\end{array}$ & $\begin{array}{l}\text { Power amplification is employed for the deprived users and power restriction for the non-deprived users. } \\
\text { Therefore, a performance trade-off can be seen between deprived and non-deprived users. By comparison } \\
\text { with selective frequency reuse, this technique is desirable only when the performance trade-off is in its } \\
\text { favor. }\end{array}$ \\
\hline $\begin{array}{l}\text { Invert } \\
\text { selective } \\
\text { power/freq. } \\
\quad \text { reuse }\end{array}$ & $\begin{array}{l}\text { It can be seen as a hybrid of selective frequency reuse and selective power reuse. This scheme focuses only } \\
\text { on the strongest interference from the neighboring cells. Basically, channel quality in cell borders can be } \\
\text { significantly increased by restricting the dominant interferer in each sub-cell (out of six) in a distributed } \\
\text { way. Compared with other reuse techniques an overall performance gain can be seen; however, the gain for } \\
\text { the deprived users is limited. }\end{array}$ \\
\hline $\begin{array}{l}\text { Self-organizing } \\
\text { channel } \\
\text { reuse }\end{array}$ & $\begin{array}{l}\text { Primarily, the macro eNBs may employ certain avoidance schemes and secondly the femtocells may overlay } \\
\text { the main network by reusing the channel in a way that does not impose excessive inter-cell interference. In } \\
\text { order to avoid potential resource collisions among macro and femto UEs, macro UEs can be ranked by the } \\
\text { macro eNB to a different frequency group. The main drawback of this technique is that it is suitable only } \\
\text { for sparse deployments since macro cell-edge UEs suffer from low SINR. Furthermore, from an operator } \\
\text { point-of-view a performance trade-off may be observed between macro and femto network in terms of } \\
\text { spectral efficiency per cell. }\end{array}$ \\
\hline
\end{tabular}

reuse is that the users at the cell borders experience worse radio conditions compared to those in the center of the cell. Therefore, it is logical to modify the spectrum to use a higher frequency reuse for the cell-edge region and a lower frequency reuse for the cell-center region. It is worth mentioning that the choice of reuse 3 for the cell-edge region has been shown to be the optimal [59]. By using this choice, the expected capacity gain can be up to $25 \%$ in OFDMA-based systems [60].

The eff. reuse (effective reuse) measure has been defined as the total bandwidth (B) divided by the bandwidth used in each cell $\left(B_{c}+B_{e}\right)$ in order to investigate the benefits of this reuse technique (this can be seen on the left in Fig. 4) [61]. For fair power distribution, the reuse 3 frequency band is amplified by factor 3 or the operational bandwidth is amplified by the eff. reuse measure. Results show that the favorable eff. reuse measure depends on the user-service policy of the packet scheduler (i.e. Max C/I or Round Robin or Proportional Fairness), which can vary it from 1.3 up to 2.0. Note that a high value is more favorable for user fairness [60] [61]. However, a major drawback of this technique is the resource underutilization in the cell-edge region since a higher reuse technique is employed.

\section{B. Interference avoidance with power division (Selective}

power reuse ${ }^{3}$ )

This variant of selective reuse technique can be seen in [62] [63], where the power transmission is amplified for users residing in the cell-edge region compared those in the cellcenter region. In addition, the selected amplified frequency group is planned orthogonally to avoid inter-cell collisions among adjacent cells. Here, to keep the total transmit power per sector fixed an amplification factor $\alpha$ is used in order to achieve the power restriction in the cell-center region and power relaxation in the cell-edge region (this can be seen on the right in Fig. 4). Similarly, the eff. reuse measure can be formulated as the ratio of the power transmissions between the cell-edge region and cell-center region i.e. $\mathrm{P}_{\text {high }} / \mathrm{P}_{\text {low }}$ [64].

By comparison with selective frequency reuse, this technique exhibits not only zero bandwidth loss but also minimizes the resource underutilization problem in the celledge region [65]. Furthermore, the performance of cell boundaries can be gradually adjusted through fine-tuning the power amplification factor ${ }^{4}$, since the selected value is not affected by the granularity of radio resources. However, no

\footnotetext{
${ }^{3}$ To avoid ambiguity of the terms selective power reuse and power control, we give the following definition. The power control is referred to as a general technique that coordinates the transmit power per RB in order to avoid excessive amount of interference across the cells. However, we refer to the selective power reuse as a technique which divides the total transmit power among the cell-edge and cell-center region to minimize the resource conflicts across different group of cells.

${ }^{4}$ Fine-tuning power amplification factor is the cell parameter that adjusts the power division between the cell-edge and cell-center region.
} 
TABle 3. General Classification OF INTER-CELl InTERFERENCE COORDINATION (ICIC)

\begin{tabular}{|c|c|c|c|}
\hline $\begin{array}{c}\text { ICIC } \\
\text { TIME SCALE }\end{array}$ & $\begin{array}{c}\text { ICIC } \\
\text { ADAPTABILITY }\end{array}$ & $\begin{array}{c}\text { ICIC } \\
\text { CLASSIFICATION }\end{array}$ & $\begin{array}{c}\text { MAIN } \\
\text { CHARACTERISTICS }\end{array}$ \\
\hline Days and more & None & Fixed (static) & Fixed network planning \\
\hline Days & $\begin{array}{l}\text { Adaptive to long-term network } \\
\text { conditions }\end{array}$ & \multirow{3}{*}{$\begin{array}{c}\text { Adaptive } \\
\text { (semi-static) }\end{array}$} & Flexible network planning \\
\hline Minutes & Cell-load adaptive & & $\begin{array}{l}\text { Flexibility on uneven traffic distributions } \\
\text { among different cells }\end{array}$ \\
\hline Seconds & User-load adaptive & & $\begin{array}{l}\text { Flexibility on user time-varying traffic } \\
\text { demand }\end{array}$ \\
\hline Milliseconds & Fully-synchronized & $\begin{array}{l}\text { Real-time } \\
\text { (dynamic) }\end{array}$ & $\begin{array}{l}\text { Effective on time-frequency channel } \\
\text { variations and to dynamic network conditions. }\end{array}$ \\
\hline $\mathrm{N} / \mathrm{A}$ & Adaptive to different type of cells & Enhanced & $\begin{array}{l}\text { Effective time-frequency resource sharing } \\
\text { among different type of cells }\end{array}$ \\
\hline
\end{tabular}

significant overall capacity gain can be seen (compared with reuse-1 system), since the interference avoidance is achieved at the expense of good channel radio conditions near the cell site [10]. As a result, in most cases only a performance tuning between the cell-edge and cell-center regions can be seen.

A comprehensive performance comparison between selective frequency reuse and selective power reuse in the regime of fixed coordination can be found in [64] [65] [66] [67]. The main trends are that the employment of a power reuse technique is beneficial to increase slightly the cell-edge throughput without jeopardizing much of the system throughput. However, for higher cell-edge throughput the employment of selective frequency reuse is a more optimal choice [68] and thus authors in [53] [54] combine both in order to provide moderate and high performance cell-edge services.

\section{Interference avoidance with dominant interference suppression (Invert selective frequency/power reuse)}

This variant of selective interference avoidance was originally reported in [70] for tri-sectorized sites and in [71] for six-sectorized sites. This idea in tri-sectorized sites is shown in Fig. 5 with seven neighboring cells. This technique can be seen as a hybrid of selective frequency reuse and selective power reuse. Compared with other techniques this scheme focuses only on the strongest interference from the neighboring cells. The key idea is to increase the channel quality to a certain frequency group $F X, X \in\{1,2, \ldots, 7\}$, in cell borders by restricting the dominant interferer in each subcell (out of six) in turn [83]. Consequently, all surrounding sectors reduce their corresponding power $\left(P_{\text {restr }}\right)$ to this certain frequency group $\overline{F X}$ leading to better radio conditions in all overlapping cell areas. To compensate for the power reduction, the remaining power is distributed on non-restricted frequency groups to the level of $P_{\text {norm }}$. The eff. reuse measure is given by the expression (7- $\beta) / 6$, where the value of $\beta$ can vary from 0 to 1 .

Interestingly, only a small number of papers have investigated the performance of this selective reuse technique. However, in this paper we provide a complete comparison of all of the techniques described above.

\section{Interference avoidance by self-organizing channel reuse for femtocells}

Femtocells are supplementary low-power eNBs that aim to extend the indoor radio coverage in a licensed band through a fixed broadband backhaul where the coverage from the main cellular network is insufficient [72]. Firstly, the macro eNBs may employ certain avoidance schemes and secondly the femtocells may overlay the main network by reusing the channel in a way that does not impose excessive inter-cell interference [73] [74]. This way of channel assignment is known as 'self-organizing' for the following reason; each femtocell may learn and self-configure itself according to its environment, integrate with the network by using available resource blocks and optimize their transmit power without interfering with the main network [75]. In order to avoid potential resource collisions among macrocells and femtocells, victim UEs in the macro area can be ranked to a different frequency group. The main drawback of this technique is that it is suitable only for sparse femto deployments since the macro cell-edge UEs are suffering from low SINR [74]. Furthermore, from an operator point-of-view a performance trade-off may be observed between macro and femto network in terms of spectral efficiency per cell. For readers interested in the area of femtocells, further interference management techniques have been surveyed recently in [77].

\section{V.CLASSIFICATION OF ICIC}

In the literature, the scale of ICIC can be classified into 
TABle 4. Classification Of ReAl-Time (DyNamic) ICIC

\begin{tabular}{|c|c|c|c|}
\hline \multirow{2}{*}{ CLASSIFICATION } & \multicolumn{3}{|c|}{ MAIN CHARACTERISTICS } \\
\hline & FRAMEWORK & PERFORMANCE & PRACTICALITY \\
\hline $\begin{array}{l}\text { Centralized or } \\
\text { global }\end{array}$ & $\begin{array}{l}\text { The problem can be modeled as an } \\
\text { optimization problem subject to a number } \\
\text { of multiple constraints. However, a fully } \\
\text { interconnected network is required. }\end{array}$ & $\begin{array}{l}\text { An optimal } \\
\text { performance as } \\
\text { compared with other } \\
\text { approaches. }\end{array}$ & $\begin{array}{l}\text { The signaling overhead and the } \\
\text { computational complexity are } \\
\text { prohibitively high for practical } \\
\text { systems. }\end{array}$ \\
\hline $\begin{array}{l}\text { Semi-centralized or } \\
\text { semi-distributed }\end{array}$ & $\begin{array}{l}\text { A significant part of the complexity of the } \\
\text { central entity is shifted to the local entities } \\
\text { of the network. }\end{array}$ & $\begin{array}{l}\text { A near-optimal } \\
\text { performance can be } \\
\text { archived. }\end{array}$ & $\begin{array}{l}\text { They are designed to cope with } \\
\text { the real-world processing load } \\
\text { and signaling delay. }\end{array}$ \\
\hline $\begin{array}{l}\text { Decentralized or } \\
\text { fully-distributed } \\
\text { (learning \& } \\
\text { unlearning, } \\
\text { supervised \& non- } \\
\text { supervised) }\end{array}$ & $\begin{array}{l}\text { No central manager, however each node is } \\
\text { allowed to exchange information and } \\
\text { perform some local processing tasks. }\end{array}$ & $\begin{array}{c}\text { Decentralized } \\
\text { solutions are based } \\
\text { on heuristic } \\
\text { algorithms and are } \\
\text { always sub-optimal. }\end{array}$ & $\begin{array}{l}\text { Highly favorable in terms of } \\
\text { inter-cell signaling and } \\
\text { complexity load. }\end{array}$ \\
\hline
\end{tabular}

three broad categories: fixed (static), adaptive (semi-static) and real-time (dynamic) [11] [35] [78]. Some general characteristics of each category are discussed below and summarized in Table 3.

\section{A. Fixed Coordination}

Fixed coordination is employed during the network planning process, mainly to mitigate inter-cell interference in the cell-edge areas. This network planning, generally, can be used for an extended period of time. In this coordination the implementation complexity and the signaling overhead is kept at minimum, since no feedback is required from the mobile terminals. However, the performance improvement is limited due to the lack of adaptability to the dynamics of the network (i.e. cell loading and user loading [35]) and to the asymmetric traffic demand (i.e. different traffic loading among cells). For comparison reasons, the performance gain archived by fixed coordination can be up to $10 \%-30 \%$ compared with the classical reuse-1 system (reference scheme) in terms of celledge throughput [60]. Further techniques based on fixed coordination can be found in [52] [63] [79], while the main trends have been reviewed in [80].

\section{B. Adaptive Coordination}

Selective reuse through adaptive coordination is generally more flexible to cope with different network characteristics such as uneven traffic distribution between cells or within a cell and therefore, yields a higher gain compared with a fixed coordination. In this category, the amount of radio resource which is dedicated to the disadvantaged wireless terminals among same/different cell groups is flexible according to different network requirements. The flexibility to different network requirements depends on the ICIC scale configured which can span from hundreds of TTIs to several days. Generally, the adaptive coordination may provide flexibility to user-load or cell-load traffic demand. Table 5 lists in detail the main differences in the time scale operation among adaptive and real-time coordination, and the variables that are sensitive to this.

In order to explain how a scheme may be adaptive in terms of cell/user loading dynamics let us consider the simple cellular model as described in section IV. By varying the eff. reuse measure of each of the abovementioned techniques across the time domain; i.e. between the cell-edge and cellcenter regions, we can adaptively satisfy the network demand within a cell. However, to react to variable traffic loading among different cell groups a reserved bandwidth portion can be lent through a request-grant mechanism [81]. This resource relaxation may impose additional signaling among cells and may even incur some instability in the RRM entity, as there is no guarantee that all requests will be granted [82]. For instance, let us consider the fixed selective frequency/power reuse cellular model. The frequency group $F 1$ from cell group 1 may request some extra RBs from frequency group $F 2$ or frequency group $F 3$, which belong to cell group 2 and cell group 3, respectively. A similar approach is demonstrated for the invert selective frequency/power reuse for the case of adaptive coordination [83].

Generally, the gain in performance is expected to be up to $10 \%-50 \%$ in average sector throughput compared with the reference scheme (reuse-1) [81] [86] [87]. Note that the adaptive approach has been introduced within the activities of 3GPP to overcome the low performance of static schemes and the high amount of inter-cell signaling required for dynamic schemes [61]. Recently the framework of adaptive coordination has been of great appeal for self-organizing 
TABLE 5. SuMmary OF X2 Signaling MECHANISMS In FACILITATING ICIC

\begin{tabular}{|c|c|}
\hline $\begin{array}{l}\text { X2 SIGNALING } \\
\text { MECHANISM }\end{array}$ & GENERAL DESCRIPTION \\
\hline RNTP & $\begin{array}{l}\text { This mechanism is mainly used for facilitating the ICIC in the DL. Each eNB can signal to the } \\
\text { neighboring eNBs on a RB basis on whether the transmit power can be higher than a certain threshold. }\end{array}$ \\
\hline HII & Each cell may inform its neighbors the future allocation set of RBs to assist the ICIC in the UL. \\
\hline IOI & $\begin{array}{l}\text { The IOI message is only triggered where severe interference is detected in the UL. } \\
\text { By signaling this, each cell can exchange several statistics for each RB based on the SINR metric. }\end{array}$ \\
\hline DL-HII & $\begin{array}{l}\text { Affected UEs can initiate this request by sending a DL-HII message to the serving outdoor eNB. Then, } \\
\text { the outdoor eNB may ask the indoor eNB to refrain from using a number of RBs indicated by this } \\
\text { message. }\end{array}$ \\
\hline
\end{tabular}

networks by self-optimizing the above network parameters [83].

\section{Real-time Coordination}

Alternatively, a real-time coordination approach can exploit the channel diversity that exists in the network more efficiently and can better adapt to any network conditions in order to achieve high coordination gains. Such schemes may operate in a timescale of a frame (TTI in the 3GPP parlance) or super-frame (several TTIs or radio frame). It is important to note that the dynamic nature of fully synchronized coordination tends to introduce high-levels of signaling overhead to the existing system and overly increases the implementation complexity and latency of the network. More generally, real-time coordination can be further classified into three subcategories according to the degree of coordination: global or centralized approach, semi-centralized approach, and decentralized approach [11]. The general characteristics of each category are discussed below and summarized in Table 4.

In global or centralized approaches, the resource coordination problem can be modeled as an optimization problem subject to a number of constraints including the level of interference, the amount of channel resource, and the number of communication terminals involved. By utilizing global channel knowledge, the problem of channel allocation through a resource restriction in the multi-cell environment is a multi-dimensional assignment problem and is proven of NPhard complexity [88] [89]. As shown in section III the complexity of the original problem can be reduced by using binary/integer LP. Similar contributions can be found in [90] [91] [92]. However, the presence of an omniscient central entity can impose computational complexity and significant signaling among cells.

In semi-centralized approaches, a significant part of the complexity of the central entity is shifted towards the BS thus they are designed to deal with the processing load and signaling delay existing in real-world systems [94]. One major advantage of this approach is that an algorithm which uses multiple discrete entities can operate on different time scales and decision levels and therefore this architecture is more practical. For example, the algorithm which resides at the central-entity decides at super frame level at which node each resource is allocated, and the algorithm which stays on a nodeentity decides at frame level to which user each resource is assigned. A popular approach of solving the interference problem in a semi-centralized manner is through an interference graph [11]. In the first phase, a heuristic algorithm may be used from Graph theory i.e. MAX k-CUT [6] [68] to indicate resource conflicts (or excessive amounts of interference). Thereafter in the second phase, an optimal channel assignment can be conducted in each BS by taking into account instantaneous channel variations.

In decentralized schemes, there is no central entity or central manager but each node is allowed to exchange information and perform some local processing tasks. However, this decentralized architecture may lead to many conflict resolutions of the same resource poll among coexisting competitors. Some popular non-cooperative framework can be adopted from game theory [95]. Despite its anarchy at early stages, it gradually aims to jointly resolve the conflicts and maximize the selfish objectives in the long-term. Eventually, a general steady state among non-cooperative selfish agents can be reached among structure, namely the Nash equilibrium [98], where each individual can achieve resource sharing without reducing its competitiveness. Some major contributions can be found in [96] [97]. However, the main challenge here is to devise a user utility function in convex form that favors inter-cell interference management in the long term. This can be achieved by formulating an intracell power control which benefits cell-edge vs. cell-centre users [99].

Recently, another popular trend in the literature is the socalled 'self-organizing' ICIC in which a decentralized algorithm, namely multi-sector gradient algorithm [100] [101] [102], pursues a flexible radio resource plan through selective frequency reuse. Despite the fact that recent developments prefer to keep the self-organizing ICIC decentralized, other works are concerned by the absence of supervision in the overall system [103]. 


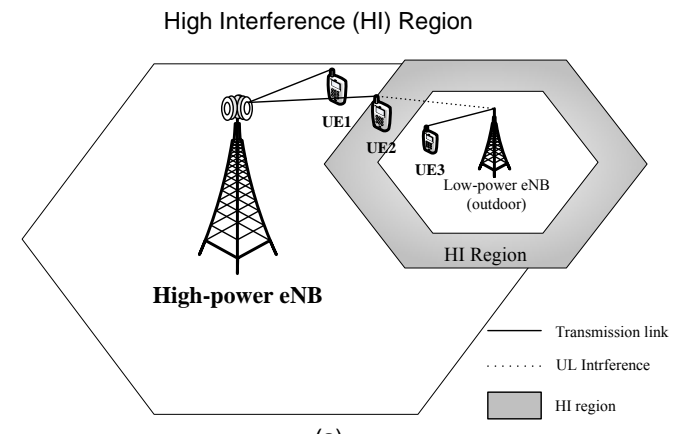

(a)

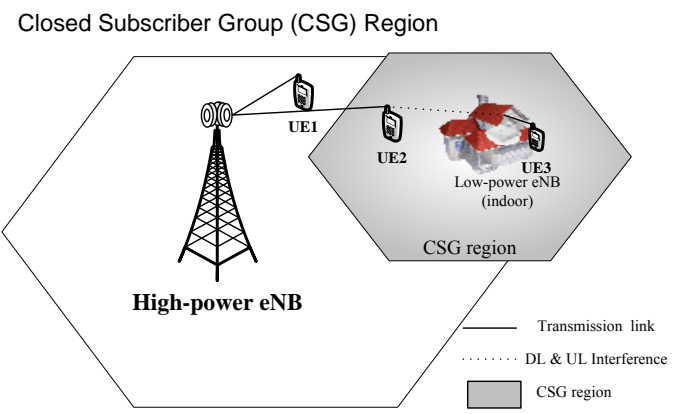

(b)

Figure 6. Two severe interference scenarios in HetNets: (a) HI region and (b) CSG region, in which the camping UEs may experience high interference from close-by low-power eNodeBs (a) or may jam nearby low-power UEs in UL (a) \& (b).

It turns out that even though the performance of these algorithms is suboptimal, the low-level of computational complexity and signaling overhead is highly favorable in practical systems. Other popular decentralized algorithms can be found in [104], i.e. learning \& non-learning, supervised \& non-supervised and non-cooperative \& cooperative.

\section{Enhanced Coordination (HetNets)}

Enhanced coordination is the interference coordination within different cell-size cellular networks where in some cases there is no direct communication interface among eNBs [109]. An example of such a scenario is a macrocell with many low-power small cells (could be indoor or outdoor). In this case, broadband providers may assist the in-between communication or this can be achieved through a dedicated wireless communication link. In either case, adaptive or real time coordination may not be possible due to the requirement of this link or it may also be overcomplicated as different cell parameters are involved. Therefore, the authors in [110] investigate some scenarios to exploit this communication link when full frequency reuse is assumed. In order to resolve the femto-to-macro interference, a message is tunneled through this communication link to a set of femtocells to restrict them from reusing frequency groups in conflict. Another interesting idea without tunneling/coordinating messages, is to detect the victim macro UE with sensing mechanisms and regulate the transmit power and radio resources [111] [112]. However, the main drawback of these self-regulated techniques is that they are very sensitive to the environmental surroundings (shadowing) near the area of the victim UE.

\section{ICIC IN EMERGING HetNet Systems}

\section{A. ICIC Facilitating Mechanisms}

3GPP LTE has standardized a variety of mechanisms to indicate and to manage the level of the inter-cell interference [105]. Initially, the first set of these standardized mechanisms are limited to the frequency/power domain only. These mechanisms are usually signaled through the $\mathrm{X} 2$ interface via a wired backbone [106] and probably via wireless channel in upcoming LTE Releases [107]. Below, we describe the X2 signaling mechanisms so-far approved and recent developments from academia and summarized in Table 5 [108]:

Relative Narrowband Transmitted Power (RNTP) This mechanism is mainly used for facilitating ICIC in the DL. Each eNB can signal to the neighboring eNBs on a RB basis whether the transmit power can be higher than a certain threshold. With this information, the neighbor eNBs may anticipate which RBs would experience a higher interference and compensate for the undesirable effect promptly without relying on the CQI reports of UEs.

High Interference Indicator (HII) - Similar to the RNTP $\mathrm{X} 2$ signaling, each cell may inform its neighbors of the future allocation set of RBs to assist the process of ICIC in the UL. In the UL, only cell-edge UEs are likely to create a highinterference scenario to the neighboring cells, thus only these UEs are indicated.

Interference Overload Indicator (IOI) - Unlike the above signaling mechanisms, the IOI message is only triggered where severe interference is detected in UL. By signaling this, each cell can exchange several statistics for each RB based on the SINR metric. With the employment of decentralized ICIC, we believe this performance update may be highly valuable for self-learning process in future TTIs.

Downlink High Interference Indicator (DL-HII) - This signaling mechanism is proposed in [110] to facilitate the RRM among outdoor and indoor eNBs assuming that a proper communication interface link exists. Affected UEs can initiate this request by sending a DL-HII message to the serving outdoor eNB. Then, the outdoor eNB may ask the indoor eNB to refrain from using a number of RBs indicated by this message.

The above-mentioned X2 signaling mechanisms are only designed to protect the data channels, thus are suitable mostly for homogeneous cell deployments. However, a range of more intelligent and promising techniques is given in future LTE releases which protect both data and control transmit symbols. 


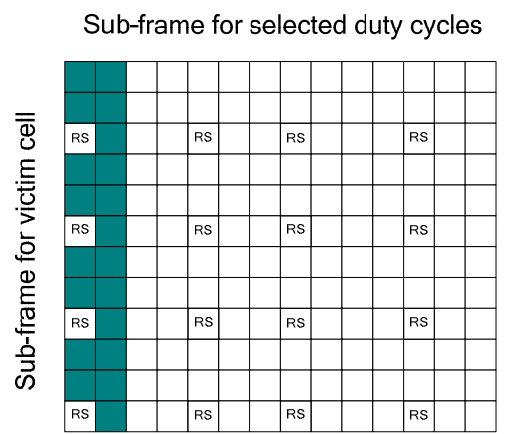

Sub-frame for remaining duty cycles
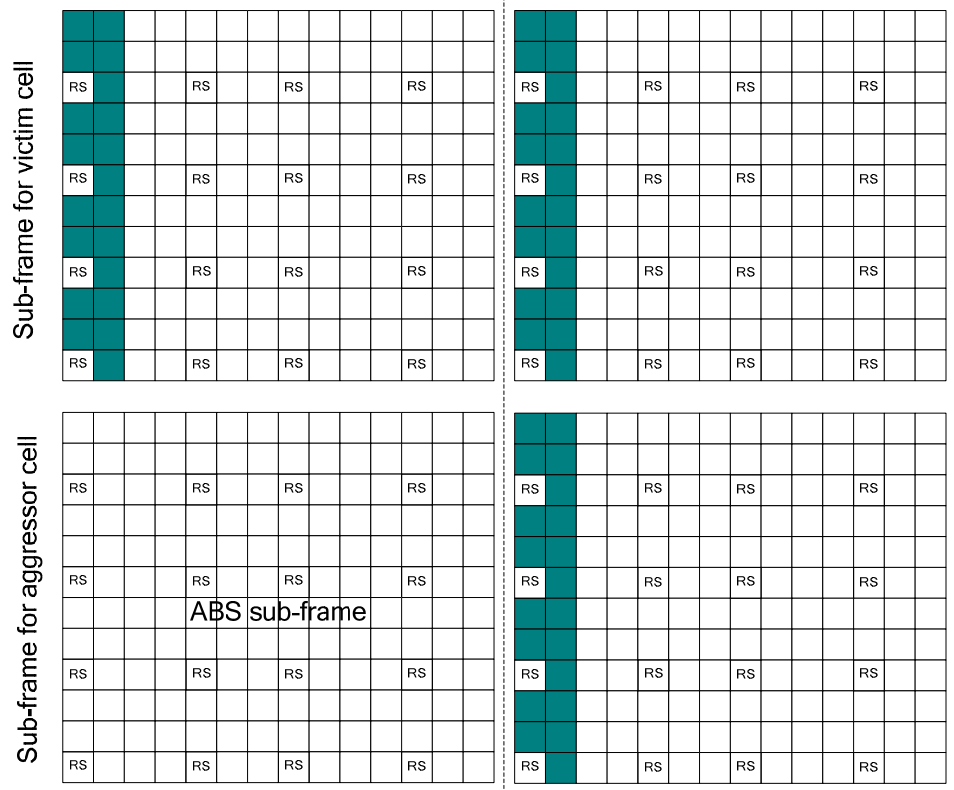

(a)
Sub-frame for all duty cycles
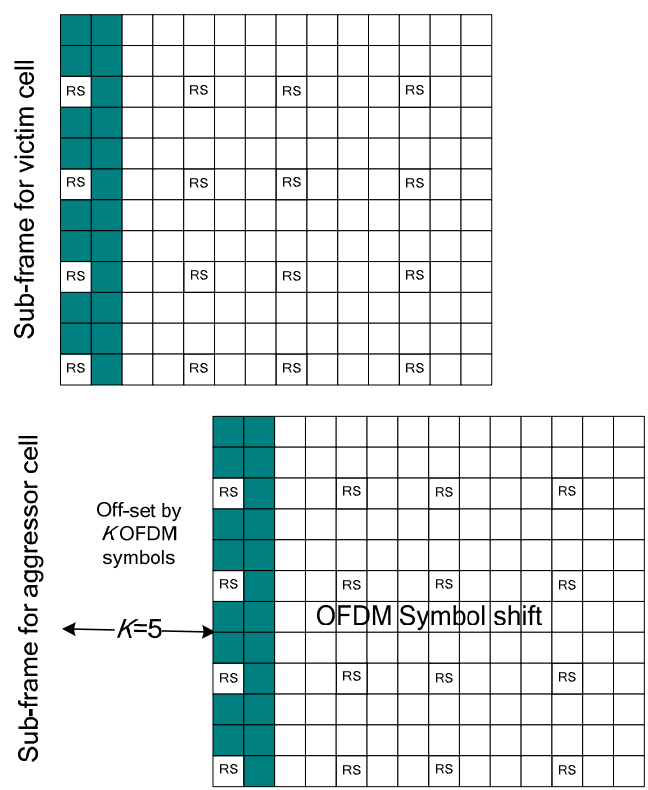

(b)

Figure 7. ABS (a) and OFDM symbol shift (b) are time-domain eICIC facilitating mechanisms to moderate the impact of large-scale HetNets. In the case of ABS technique, the sub-frame of the aggressor cell remains silent or mute for a number of selected duty cycles. In this period, the victim cell may experience a lessened interference. However, in the case of OFDM symbol shift technique the sub-frame alignment of aggregator cell may be shifted by a number of OFDM symbols. However, this offset is favorable if most of the control and reference symbols do not collide. For the legend, refer to Fig. 2.

\section{B. Severe Interference Scenarios in HetNets}

With the proliferation of low-power eNBs -either indoor or outdoor - within the macro coverage, some interference scenarios start to become known and more obvious. To deal with this new interference sources, a set of more sophisticated techniques is needed [114]. Fig. 6 illustrates two major interference scenarios that can exist in a typical HetNet and these are briefly discussed below:

High Interference (HI) region - This severe uplink interference scenario is observable due to the large power difference between nodes. It is evident that in a HetNet a cellselection process based on the DL Reference Symbols (RSs), where their transmit power can vary from node to node, may not be the best strategy since there is some undesirable intercell interference. Firstly, it only minimizes the inter-cell interference in DL and secondly, it tends to overload the nodes with high-transmit power. Therefore, a more small-cell aware cell-selection procedure is immediately required to handover these UEs from the HI region. One possible solution is through an appropriate offset that can be added to low-power eNBs' DL RSs [116]. With this offset, the HI region can be converted to a range-expanded region [114] so these interfering UEs can now be members of this small cell. However, this novel procedure may result in poor reception in DL, since these new UEs are not served by the nearest cell [115]. To this end, we believe that a further investigation is necessary on this dubious region.

In a closed subscriber group (CSG) region severe interference originates from low-power nodes that may be deployed in a domestic area and provide only CSG services. In a domestic environment, non-CSG members experience poor coverage due to wall penetration and strong indoor interference. As a result, these UEs are normally associated with high-transmit power in UL, which can affect severely the services of nearby CSG nodes. To overcome this issue, nonCSG members can be temporally accommodated to this subscriber group, subject to a number of restrictions in the provided service.

\section{C. eICIC techniques}

A range of more promising ICIC techniques is given in LTE-A, well known as eICIC, in order to protect both the control and data channel information. We briefly describe enhanced ICIC (eICIC) techniques that have been adopted by 3 GPP.

\section{1) Time Domain}

In a typical OFDMA mobile network, all radio resource units are mainly considered time aligned. Depending on this alignment, different time-domain techniques may be deployed to handle interference. Fig. 7 provides a sketch of radio resource units in the DL of two typical techniques that may be used in the case of two cells in conflict, i.e. aggressor and victim. A further explanation of these techniques is given below:

Almost Blank Subframe (ABS) - At a given duty cycle, ABS may be used to protect the synchronized sub-frames 
TABLE 6. SuMmARY OF ENHANCED-ICIC (EICIC) TECHNIQUES CONSIDERED IN HETNETS

\begin{tabular}{|c|c|c|}
\hline \multicolumn{2}{|c|}{$\begin{array}{l}\text { AVOIDANCE } \\
\text { TECHNIQUE }\end{array}$} & GENERAL DESCRIPTION \\
\hline \multirow{2}{*}{$\begin{array}{l}\text { Time } \\
\text { domain }\end{array}$} & ABS & $\begin{array}{l}\text { The ABS time-domain technique may be used for a selected duty cycle in order to reduce the } \\
\text { interference from the aggregator cell to the victim cell. In this subframe, only RSs are transmitted } \\
\text { whereas control or data symbols are muted. }\end{array}$ \\
\hline & $\begin{array}{l}\text { OFDM } \\
\text { symbol } \\
\text { shift }\end{array}$ & $\begin{array}{c}\text { The scheduling block between aggregator-victim cells may be shifted by K OFDM symbols. } \\
\text { Compared with ABS technique the scheduling blocks are active in all time transmission intervals but } \\
\text { are not aligned. }\end{array}$ \\
\hline \multicolumn{2}{|c|}{$\begin{array}{l}\text { Frequency } \\
\text { domain }\end{array}$} & $\begin{array}{l}\text { A fully orthogonal channel is provided to the conflicted cells at the cost of reduced operational } \\
\text { bandwidth }\end{array}$ \\
\hline \multicolumn{2}{|c|}{ Power domain } & $\begin{array}{l}\text { A power control technique may be used among different type of cells when the interference radiated } \\
\text { exceeds a measured threshold. }\end{array}$ \\
\hline
\end{tabular}

between victim and aggressor cells. In this subframe, the aggressor cell remains silent or mute by transmitting an empty cell for a number of selected duty cycles. In this way, the victim cell may experience a lessened interference since the control and data signals are muted (not transmitted). In the remaining duty cycle, normal sub-frames take place.

OFDM Symbol Shift - In OFDM Symbol Shift technique, the sub-frame of the victim cell is shifted by $K$ OFDM symbols with respect to the sub-frame of the aggressor. For example, the choice of $K=3$ or $K=5$ (Fig, 7) shows much interest in order to avoid collisions with all the control and most reference symbols. In conjunction with this, the above ABS technique can be used to further reduce the inter-cell interference.

Generally, time-domain eICIC techniques are highly dependent on the sub-frame synchronization across the network. Given that currently conventional OFDMA systems are quite susceptible to time synchronizing errors [118], such a consideration for the emerging HetNets is a major challenge, taking into account that the network backhaul may be a mixture of wired (leased \& not leased) and wireless technologies [117].

\section{2) Frequency Domain}

In contrast with the time domain, the transmission of physical signals (including synchronization symbols) and control channels in frequency-domain eICIC can be completely orthogonal among cells in conflict, at the cost of a reduced bandwidth [7]. Similarly, to increase the spectral reuse efficiency this can be individually performed in a dynamic manner when a victim UE is detected.

\section{3) Power Domain}

Another approach to reduce inter-cell interference is through power control. Although the total downlink power control is not considered for the outdoor eNBs, a power control technique is expected to be employed in indoor lowpower nodes to further mitigate inter-cell interference. For this reason, transmit power of an aggressor cell may be reduced in the case that it exceeds a predefined fixed threshold at the victim UEs, in scenarios where nearby macro UEs are located close to these premises. However, restricting the maximum transmit power of the low-power nodes may hamper significantly femtocell overall performance. The authors in [7] investigate the performance and computational complexity of different power control techniques using different threshold based metrics. The eICIC techniques considered in paper are summarized in Table 6.

\section{Main Challenges ANd Future Evolution of ICIC}

\section{A. Carrier aggregation}

Carrier aggregation has been recently employed in LTE-A in order to extend the total operational bandwidth. This can be achieved by operating concurrently up to five licensed carriers of $20 \mathrm{MHz}$ each. However due to different features that each new LTE release brings, three different compatibility modes are envisioned: backward-compatible, non-backwardcompatible and extension. In few words, the backwardcompatible mode can be accessed from any UE regardless of supported LTE release, whereas the non-backward-compatible mode can be accessed only from LTE-A UEs. The extension mode can be employed by any UE in order to extend another counterpart as a part of the carrier aggregator set. In aggregating these carriers, two possible scenarios are feasible: contiguous and non-contiguous. The latter scenario can be aggregated either in a single band or over multiple bands [119].

Due to above possible accessibility modes and aggregating scenarios, different component carriers can be used and configured differently. In addition, different ratios between DL and UL carriers can be assigned to a single UE. This multi-band implementation flexibility across all the aggregate carriers introduces diversity in network coverage and widespread service differentiation. Therefore, this asymmetry brings a lot of challenges for future ICIC techniques in order to protect both the data and control channels in an emerging 


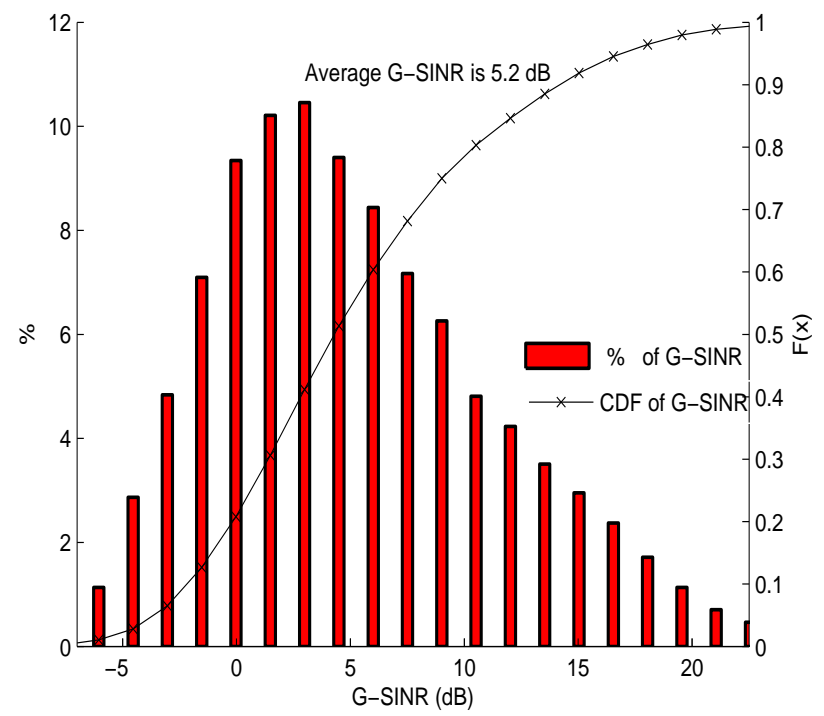

Figure 8. Histogram and CDF of geometry SINR (G-SINR) of a 2-tier LTEbased system with tri-sectorized antennas in an urban environment.

\section{LTE-A HetNet.}

\section{B. Self-Organizing ICIC}

As discussed earlier, the framework of adaptive coordination is considered favorable for self-organizing networks by self-optimizing the cell loading and user loading network parameters. Furthermore, the amount of RBs, which are dedicated for the deprived region, may be subject to optimization. An interesting simple approach is to hibernate eNBs not in use in order to reduce the transmit interference to the other cells. Nevertheless, the design of a large selforganizing network becomes complicated to deal with, with the increased number of system parameters and with the uneven traffic demand across different type of cells [7].

Another approach is through decentralized ICIC algorithms or algorithms which require a minimum supervision. In conjunction with different self-organizing techniques, their cooperation can be independent with minimum information exchange while aiming to increase the overall network performance in the long-term. Considering the self-organizing framework, a self-organizing ICIC has to be scalable, stable and agile [104]. An agile algorithm means that the algorithm is capable of coping with the dynamics of the network and treats each user independently according to its requirements. However, in order to make our algorithm scalable, the original problem may be divided into master and slave sub-problems. Since, our problem belongs in LP, a number of ways exist in the literature such as primal and dual decomposition [120]. Here, the master problem may be solved using a projected subgradient method or another stable iterative method.

This approach becomes a necessity in HetNets where indoor nodes can be deployed in an ad hoc manner and are subject to movement or switched on/off at any time. To further emphasize this requirement, most conventional network
TABle 7. Main System SimUlation PARAMETERS

\begin{tabular}{|c|c|}
\hline PARAMETER & VALUE \\
\hline Deployment Scenario & $\begin{array}{l}\text { 2-tier tri-sectorized sites } \\
\text { (19-sites, } 57 \text { eNodeBs) }\end{array}$ \\
\hline Inter-Site Distance (ISD) & $500 \mathrm{~m}$ \\
\hline Total Bandwidth & $10 \mathrm{MHz}$ \\
\hline No of Resource Blocks (RB) & $100 \mathrm{RBs}$ \\
\hline Total bandwidth per RB & $180 \mathrm{kHz}$ \\
\hline eNB Power & $43 \mathrm{dBm}$ \\
\hline Path loss model & $\mathrm{L}=128.1+37.6 \log _{10}(\mathrm{R}), \mathrm{R}[\mathrm{KM}]$ \\
\hline $\begin{array}{c}\text { Shadowing's } \\
\text { standard deviation }\end{array}$ & $8 \mathrm{~dB}$ \\
\hline $\begin{array}{l}\text { Shadowing's } \\
\text { correlation distance }\end{array}$ & $50 \mathrm{~m}$ \\
\hline Channel model & Extension of ITU Pedestrian B \\
\hline Moving Speed & $3 \mathrm{Km} / \mathrm{h}$ \\
\hline Antenna mode & SISO \\
\hline Antenna gain model & Berger \\
\hline User Noise figure & $9 \mathrm{~dB}$ \\
\hline Scheduling Algorithm & Proportional fairness (PF) \\
\hline PF averaging time window & 25 TTIs \\
\hline Traffic model & Full buffer \\
\hline Num of UEs per sector & 10 \\
\hline Simulation time in each drop & 100 TTIs \\
\hline Total number of drops & 100 \\
\hline
\end{tabular}

planning/optimization tools can easily meet a processing bottleneck when taking into account a large number of lowpower nodes.

\section{Energy-Efficient ICIC}

A recent analysis has shown that the average energy consumption in cellular basestations can reach up to $60 \%$ of the total network energy consumption [121]. Although energy consumption has become more apparent in the literature, only a few papers have addressed the energy efficiency and the interference mitigation of a wireless network together. In a similar way, to minimize the energy consumption and to increase the cell-edge throughput in multi-cell cellular networks, energy-efficient ICIC schemes are required.

Interestingly, by applying interference avoidance to a number of interferers, less power is transmitted, to some extent higher spectral efficiency can be seen, and thus higher energy efficiency is observed. In order to further minimize the power consumption across the network an intra-cell power control may be employed afterwards. However, implementing an energy-efficient ICIC is an intricate task due to the novel metric that needs to be considered, i.e. bits-per-joule [122] rather than bits-per-Hz. In this case, another set of restrictions may be applied in order to maximize the energy efficiency. Back to our ICIC formulation, an appropriate utility function is needed to capture all the inter-cell energy saving stemming 


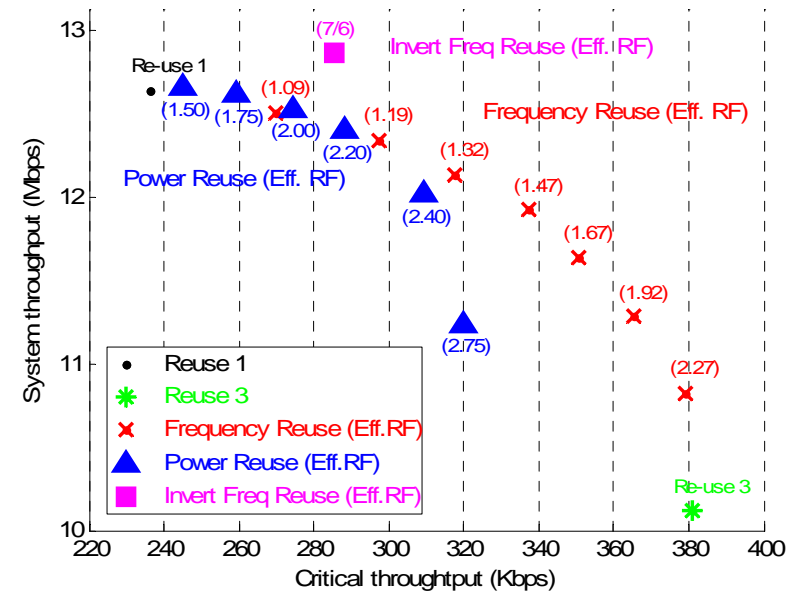

Figure 9. The system throughput is depicted against the throughput of deprived UEs (critical throughput) for all the some baseline reuse techniques using a varying choice of effective reuse measure.

from employing interference avoidance. This can include also the circuit and the modulation/demodulation energy consumption. Therefore, we feel that further examination is required in reducing the network operating costs as well as in addressing any environmental issues.

\section{SimULATION STUDY}

The simulation study is performed on the downlink using the freely available LTE-based system-level simulator [124] in order to evaluate the performance of the state-of-the-art schemes at which full or selective avoidance may be employed. Since the existing platform is based on classical frequency reuse no benchmark schemes are employed, these are implemented for the purposes of this paper. For system calibration purposes, the distribution of geometry SINR (GSINR) in terms of CDF and PDF of the simulated system is shown in Fig. 8. Here, a typical 2-tier scenario is assumed, which is served by 19 tri-sectorized sites with $500 \mathrm{~m}$ as intersite distance. Although the simulation is performed across the whole network, the performance results are taken from the central site, in which two tiers of interference are experienced. The rest of the simulation parameters are given in Table 7.

For implementation simplicity, all investigated techniques are currently in fixed form. However, a varying choice of eff. reuse measure is considered to examine their performance. The invert power/freq reuse can be either invert power reuse or invert frequency reuse according the choice of eff. reuse measure. As the latter performed best, only these results are presented. We observe the average sector throughput as the system performance and the throughput of deprived UEs as the critical performance. Therefore, to capture the throughput of deprived UEs, a common metric is used, i.e. the $5^{\text {th }}$ percentile point of CDF of UE throughput [11]. Although long-term SINR is of great interest in illustrating the problematic areas suffering from low coverage, it associates a particular geographic location with a fixed performance value [125]. Such an area-dependent metric has no channelawareness and thus no scheduling gain is observed from exploiting the diversity of the channel. Furthermore, we use a utility measure similar to proportional fairness to identify the deprived UEs [126]. For fair comparison among all the employed techniques, the total transmit power per eNB is fixed and the same across the entire simulation time.

The performance comparison in throughput of state-of-theart, based on the above-mentioned performance metrics, is presented in Fig. 9. To avoid ambiguity and enable the reader to clarify each others' performance, each of the evaluated schemes is associated with a specific marker and color as indicated in the figure legend. Since many schemes are evaluated from the same reuse technique, each performance point on the figure is linked with a certain eff. reuse measure. For convenience in overall performance, we display the system and critical performance in the same figure. With this illustration, schemes which are located at the top-right corner are more favorable, and schemes which are located in the bottom-left corner are less desirable.

As expected, the classical frequency reuse (reuse-1) scheme shows minimum cell-edge performance since no reuse technique is employed. On the other hand, the full frequency reuse 3 (reuse-3) exhibits superior critical performance by sacrificing the system throughput. The superior critical performance of reuse- 3 is attributed to suppression of strong inter-cell interference across all UEs. It can be seen from the figure that selective frequency/power reuse schemes have a performance tradeoff between system and critical throughput as the eff. reuse measure increases over a given range. It can also be observed that the critical performance of the selective frequency reuse scheme is limited by the reuse-3.

Here, we can see similar observations in performance to some of the work in the literature, as discussed in Section IV. As anticipated, the interference avoidance gain achieved with selective power reuse is more effective for small improvement of the cell-edge performance. This can be seen when the selective power reuse (2.00) is compared with selective frequency reuse (1.09). However, to achieve higher critical performance the selective frequency reuse is more desirable; readers may refer to selective power reuse (2.75) vs. selective frequency reuse (1.32).

Looking into the last scheme that employs a selective invert frequency reuse, an overall gain at both system and critical throughput can be observed. Surprisingly, the increase of the critical performance does not reduce the system throughput. This performance gain in both metrics (system and critical) is due to the suppression of the dominant interference. As a result, a higher gain in throughput is achieved through appropriate resource restrictions. However, it cannot reach the critical performance of the selective frequency/power reuse. 
TABLE 8. SUMmary OF Future EVOLUTION OF ICIC

\begin{tabular}{|c||c|c||}
\hline DIRECTION & GENERAL DESCRIPTION \\
\hline \hline $\begin{array}{c}\text { ICIC with Carrier } \\
\text { Aggregation }\end{array}$ & $\begin{array}{c}\text { A number of licensed carriers can be merged in order to extend the total operational bandwidth. } \\
\text { However due to different features that each new LTE Release brings, some compatibility modes are } \\
\text { envisioned and some aggregating scenario are considered. Therefore, this diversity brings many } \\
\text { challenges for future ICIC techniques in order to protect both the data and control channels in an } \\
\text { emerging LTE-A HetNet. }\end{array}$ \\
\hline \hline $\begin{array}{c}\text { Self-Organizing } \\
\text { ICIC }\end{array}$ & $\begin{array}{c}\text { Recently the framework of adaptive and self-organizing network has been of great appeal for self- } \\
\text { organizing ICIC. The large-scale of indoor or outdoor low-power nodes motivates the need for } \\
\text { algorithms requiring minimum supervision. However, in order to make our algorithm scalable, the } \\
\text { original problem may be distributed into master and slave sub-problems. The challenge here is to } \\
\text { maintain all the self-organizing properties within the ICIC i.e. stability, agility, scalability. }\end{array}$ \\
\hline \hline Energy-efficient \\
ICIC & $\begin{array}{c}\text { Implementing an energy-efficient ICIC is an intricate task due to the novel metric that needs to be } \\
\text { considered, i.e. bits-per-joule rather than bits-per-Hz. Back to our ICIC formulation, an appropriate } \\
\text { utility function is needed to capture all the energy saving stemming from employing interference } \\
\text { avoidance. For that reason, we feel that further examination is required in reducing the network } \\
\text { operating costs as well as in addressing any environmental issues. }\end{array}$ \\
\hline \hline
\end{tabular}

\section{CONCLUSION}

This paper provides a clear explanation of the concept of interference avoidance through inter-cell interference coordination (ICIC) for emerging multi-cell OFDMA-based wireless networks. Furthermore, a complete performance comparison of state-of-the-art selective interference avoidance is given. By and large, interference avoidance techniques can be used by applying different power-frequency restrictions in order to increase the critical performance of the cell.

Numerous publications have highlighted the need for efficient ICIC techniques in OFDMA networks, while the binary solution is still a challenge for large-scale systems. However, in this paper we describe through a series of steps how the complexity associated with the ICIC problem can be simplified and solved in polynomial time. Thus, it is highly desirable to further investigate efficient interference avoidance techniques through low-complexity ICIC that enhance both the overall and critical performance of the system. We declare that there is still an on-going research effort to find less complex and more efficient centralized ICIC employing these techniques.

Recently, a few new areas that can be seen as a future evolution of ICIC have begun to draw attention, e.g. carrier aggregation, self-organization and energy efficiency. Even though such future aspects seem very promising in the emerging HetNets, only a limited number of studies have fully addressed this issue. To the best of our knowledge, we have provided an extensive list of references, we have discussed many important issues, and we have given future directions and challenges (which are summarized in Table $8 \& 9$ ) for those readers interested in this progressively growing area.

\section{ACKNOWLEDGMENT}

This work has been performed in the framework of the ICT project ICT-4-248523 BeFEMTO, which is partly funded by the European Union. The authors would like to acknowledge the contributions of their colleagues from the BeFEMTO consortium.

\section{APPENDIX A: NOMENCLATURE}

Table 10 explains the abbreviations used in this paper.

\section{REFERENCES}

[1] 3GPP TR 36.814, "Further advancements for E-UTRA physical layer aspects" v.9.0.0, www.3gpp.org, May 2010.

[2] T. Kwon, H. Lee, S. Choi, J. Kim, D. H. Cho, S. Cho, S. Yun, W. H.Park, K. Kim, "Design and implementation of a simulator based on a cross-layer protocol between MAC and PHY layers in a WiBRO compatible IEEE 802.16e OFDMA system", IEEE Communation Magazine, vol. 43, no. 12, pp. 136-146, Dec. 2005.

[3] Xin Wang, R. Gharpurey, "Interference Cancellation in Broadband Wireless Systems Utilizing Phase-Aligned Injection-Locked Oscillators", IEEE Transactions on Circuits and Systems II: Express Briefs, vol.55, no.9, pp.872-876, Sep. 2008.

[4] F. Gomez-Cuba, R. Asorey-Cacheda, F. Gonzalez-Castano, "A Survey on Cooperative Diversity for Wireless Networks", IEEE Communications Surveys \& Tutorials, vol.13, no.99, pp.1-14, $3^{\text {rd }}$ Quarter 2011

[5] J. Mietzner, R.Schober, L.Lampe, W.H. Gerstacker, P.A. Hoeher, "Multiple-antenna techniques for wireless communications - a comprehensive literature survey", IEEE Communications Surveys \& Tutorials, vol.11, no.2, pp.87-105, $2^{\text {nd }}$ Quarter 2009.

[6] R.Y. Chang, Z. Tao, J. Zhang, C.-C.J. Kuo, "Multicell OFDMA downlink resource allocation using a graphic framework," IEEE Transaction on Vehicular Technology, vol.58, pp.3494-3507, Sep. 2009.

[7] D. Lopez-Perez, I. Guvenc, G. de la Roche, M. Kountouris, T. Q. S. Quek, J. Zhang, "Enhanced inter-cell interference coordination challenges in heterogeneous networks", IEEE Wireless Communications, vol.18, no.3, pp.22-30, Jun 2011.

[8] V. H. MacDonald, "The cellular concept", Bell System Technical Journal, vol.58, no., pp.15-41, Jan. 1979. 
TABle 9. Summary Of The Main Design Challenges Associated With ICIC

\begin{tabular}{|c|c|c|}
\hline \multicolumn{2}{|c|}{$\begin{array}{l}\text { CHALLENGES \& } \\
\text { OPEN PROBLEMS }\end{array}$} & GENERAL DESCRIPTION \\
\hline \multicolumn{2}{|c|}{$\begin{array}{l}\text { Optimal cell- } \\
\text { specific reference } \\
\text { sequences }\end{array}$} & $\begin{array}{l}\text { Cell-specific reference sequences (RS) are pilot information essential for tracking and estimating } \\
\text { desired/interfering cells. In order to enable the interoperability of different type of cells, pilot } \\
\text { sequences is one of the more important issues that operators must deal with before these networks } \\
\text { become widely deployed. Furthermore, the orthogonality of the cell-specific reference sequences can } \\
\text { be viewed as a key requirement for the functionality of dynamic ICIC techniques. }\end{array}$ \\
\hline \multirow{5}{*}{ 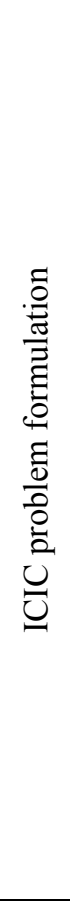 } & $\begin{array}{l}\text { Converting } \\
\text { ICIC into } \\
\text { convex form }\end{array}$ & $\begin{array}{l}\text { In order to reduce the complexity involved with the scalability of the problem we may reformulate } \\
\text { some functions into convex form. For example, the convexity of the ICIC problem can be improved by } \\
\text { redefining the operating rate function by assuming a log-transformed rate formulation. Furthermore, } \\
\text { the complexity of the original problem can be significantly reduced by adopting a property called total } \\
\text { unimodularity, which have small implications to the original problem. }\end{array}$ \\
\hline & $\begin{array}{l}\text { Converting } \\
\text { ICIC into a } \\
\text { MCNF } \\
\text { problem }\end{array}$ & $\begin{array}{l}\text { Solving a MCNF problem can be quicker than a generic LP solver. The mitigation of two or more } \\
\text { dominant interfering eNBs through a MCNF formulation has not been investigated yet. In future } \\
\text { dense-mobile environments such as femtocells, the mitigation of more than one dominant interfering } \\
\text { eNBs is essential. }\end{array}$ \\
\hline & $\begin{array}{l}\text { Distributed } \\
\text { approach }\end{array}$ & $\begin{array}{l}\text { Another way to reduce the computational complexity is to divide the original problem into a number of } \\
\text { smaller sub-problems (i.e. network problem and local problem) in order to deal with the processing } \\
\text { load and signaling delay which exist in real-world systems. A number of decomposition methods may } \\
\text { be used from optimization theory in the literature to reformulate the problem into single-master and } \\
\text { multiple-slave sub-problems. }\end{array}$ \\
\hline & $\begin{array}{c}\text { Low- } \\
\text { complexity } \\
\text { sub-optimal } \\
\text { solutions }\end{array}$ & $\begin{array}{l}\text { It is highly desirable to investigate efficient interference avoidance techniques through low-complexity } \\
\text { ICIC that enhances both the overall and critical performance of the system. We declare that there is } \\
\text { still an on-going research effort in finding less complex and more efficient near optimal algorithm. }\end{array}$ \\
\hline & $\begin{array}{l}\text { Effective } \\
\text { cutting plane } \\
\text { algorithms }\end{array}$ & $\begin{array}{l}\text { Binary/integer based problem may lead to an extensive search path in finding the optimal solution. A } \\
\text { prolonged extensive search of such a problem can be reduced significantly by introducing a number of } \\
\text { tighter constraints or cuts. However, generating cuts can be a dubious step since it may increase the } \\
\text { size of the original problem without removing significant search paths. }\end{array}$ \\
\hline \multicolumn{2}{|c|}{$\begin{array}{l}\text { Enhanced } \\
\text { Coordination \& } \\
\text { Victim UE detection }\end{array}$} & $\begin{array}{l}\text { An interesting idea for minimizing the femto-to-macro interference is to detect the victim macro UE } \\
\text { with sensing mechanisms so the aggregator femtocell can regulate the transmit power and radio } \\
\text { resources. However, the main drawback of these self-regulated techniques is that they are very } \\
\text { sensitive to the environmental surrounding (shadowing) near the area of the victim UE. }\end{array}$ \\
\hline \multicolumn{2}{|c|}{$\begin{array}{l}\text { Range } \\
\text { expansion }\end{array}$} & $\begin{array}{l}\text { A novel cell-selection procedure is introduced in LTE-A to minimize the interference arriving from a } \\
\text { large-scale small-cell deployment. This novel procedure may result in poor reception in DL, since } \\
\text { these new UEs are not served by the nearest cell. To this end, we believe that a further investigation is } \\
\text { necessary on this. }\end{array}$ \\
\hline \multicolumn{2}{|c|}{$\begin{array}{l}\text { Closed subscriber } \\
\text { group (CSG) }\end{array}$} & $\begin{array}{l}\text { Closed subscriber group (CSG) networks may be deployed in a domestic area and provide only CSG } \\
\text { services resulting in strong indoor interference to non-CSG users. A further analysis is needed, how the } \\
\text { non-CSG users can be temporally accommodated to this subscriber group. }\end{array}$ \\
\hline \multicolumn{2}{|c|}{$\begin{array}{c}\text { Sub-frame } \\
\text { synchronization }\end{array}$} & $\begin{array}{l}\text { Time-domain eICIC techniques are highly dependent on the sub-frame synchronization across the } \\
\text { network. Considering that the network backhaul may be a mixture of wired (leased \& not leased) and } \\
\text { wireless technologies, avoiding the synchronizing errors in an emerging HetNets is a major challenge }\end{array}$ \\
\hline \multicolumn{2}{|c|}{$\begin{array}{l}\text { Power control } \\
\text { techniques for low- } \\
\text { power eNBs }\end{array}$} & $\begin{array}{l}\text { Power control techniques may be employed by indoor eNBs to further mitigate inter-cell interference } \\
\text { between macro and pico/femto cells. However, is not immediately clear the number of network } \\
\text { parameters needed to be accounted for. }\end{array}$ \\
\hline \multicolumn{2}{|c|}{$\begin{array}{l}\text { Orthogonal } \\
\text { allocation } \\
\text { channels }\end{array}$} & $\begin{array}{l}\text { In order to mitigate the inter-cell interference a number of completely orthogonal channels may be } \\
\text { considered for the low-powered eNBs for certain scenarios. Since this comes at the cost of a reduced } \\
\text { bandwidth, a threshold parameter may be optimized for specific scenarios. Moreover, to increase the } \\
\text { spectral reuse efficiency this can be individually performed in a dynamic manner when a victim UE is } \\
\text { detected. }\end{array}$ \\
\hline
\end{tabular}


[9] R. Kwan, C. Leung, "A Survey of Scheduling and Interference Mitigation in LTE", Journal of Electrical and Computer Engineering, vol., no., pp., 2010.

[10] G. Boudreau, J. Panicker, N. Guo, R. Chang, N. Wang, S. Vrzic, "Interference coordination and cancellation for 4G networks", IEEE Communications Magazine, vol.47, no.4, pp.74-81, Apr. 2009.

[11] M. Necker, "Interference Coordination in Cellular OFDMA Networks", IEEE Network, vol.22, no.6, pp.12-19, Dec. 2008.

[12] M. Shariat, A. Quddus, S. Ghorashi, R. Tafazolli, "Scheduling as an important cross-layer operation for emerging broadband wireless systems", IEEE Communications Surveys \& Tutorials, vol.11, no.2, pp.74-86, 2nd Quarter 2009.

[13] D. Leith, V. Subramanian, K. Duffy, "Log-convexity of rate region in 802.11e WLANs", IEEE Communications Letters, vol.14, no.1, pp.5759, Jan. 2010.

[14] S. Sadr, A. Anpalagan, K. Raahemifar, "Radio Resource Allocation Algorithms for the Downlink of Multiuser OFDM Communication Systems", IEEE Communications Surveys \& Tutorials, vol.11, no.3, pp.92-106, 3rd Quarter 2009.

[15] M. Salem, A. Adinoyi, M. Rahman, H. Yanikomeroglu, D. Falconer, Y.D. Kim, E. Kim, Y.-C. Cheong, "An Overview of Radio Resource Management in Relay-Enhanced OFDMA-Based Networks", IEEE Communications Surveys \& Tutorials, vol.12, no.3, pp.422-438, $3^{\text {rd }}$ Quarter 2010.

[16] M.J. Marsan, G.C. Hess, S.S. Gilbert, "Shadowing variability in an urban land mobile environment at $900 \mathrm{MHz}$ ", Electronics Letters, vol.26, no.10, pp.646-648, May 1990.

[17] M. Patzold, Mobile Fading Channels, John Wiley \& Sons, Inc., New York, USA, pp.33, 2002.

[18] Y. R. Zheng, C. Xiao, "Simulation models with correct statistical properties for Rayleigh fading channels", IEEE Transactions on Communications, vol.51, no.6, pp.920-928, Jun. 2003.

[19] A. Paulraj, R. Nabar, D. Gore, Introduction to Space-Time Wireless Communications, Cambridge University Press, Cambridge, UK, 2003.

[20] J. S. Wang, J. H. Lee, J. C. Park, I. Song, Y. H. Kim, "Combining of Cyclically Delayed Signals: A Low-Complexity Scheme for PAPR Reduction in OFDM Systems", IEEE Transactions on Broadcasting, vol.56, no.4, pp.577-583, Dec. 2010.

[21] E. Dahlman, S. Parkvall, J. Skold, P. Beming, "3G Evolution: HSPA and LTE for Mobile Broadband", $2^{\text {nd }}$ Ed., Academic Press, pp.327, Oxford, UK, 2008.

[22] 3GPP TR 36.211, "Evolved Universal Terrestrial Radio Access (EUTRA); Physical channels and modulation" v.10.4.0, www.3gpp.org, Dec. 2011.

[23] K. Rajeswari, T. Sangeetha, A.P. M. Natchammai, Nandhini, S. J. Thiruvengadam, "Performance analysis of pilot aided channel estimation methods for LTE system in time-selective channels", 5th International Conference on Industrial and Information Systems (ICIIS), vol., no., pp.113-118, Aug. 2010.

[24] Sesia, Toufik, Baker, LTE - The UMTS Long Term Evolution: From Theory to Practice, Wiley, pp.163, 2009.

[25] J. Won Kang, Y. Whang, H. Y. Lee, K. S. Kim, "Optimal Pilot Sequence Design for Multi-Cell MIMO-OFDM Systems", IEEE Transactions on Wireless Communications, vol.10, no.10, pp.33543367, Oct. 2011.

[26] Yu Wei, J. M. Cioffi, “Constant-power waterfilling: performance bound and low-complexity implementation", IEEE Transactions on Communications, vol.54, no.1, pp.23-28, Jan. 2006.

[27] J. Kim, D.-H. Cho, "A Joint Power and Subchannel Allocation Scheme Maximizing System Capacity in Indoor Dense Mobile Communication Systems", IEEE Transactions on Vehicular Technology, vol.59, no.9, pp.4340-4353, Nov. 2010.

[28] A. Simonsson, "Frequency Reuse and Inter-cell Interference CoOrdination In E-UTRA", IEEE 65th Vehicular Technology Conference, (VTC-Spring), vol., no., pp.3091-3095, Apr. 2007.

[29] R. Kwan, C. Leung, "A Survey of Scheduling and Interference Mitigation in LTE", Journal of Electrical and Computer Engineering, vol., no., pp., 2010.

[30] P. Godlewski , M. Maqbool, M. Coupechoux, J. Kélif, “Analytical evaluation of various frequency reuse schemes in cellular OFDMA networks". 3rd International Conference on Performance and Evaluation Methodologies and Tools, Athens, Greece, Oct. 2008.

[31] M. Rahman, H. Yanikomeroglu, "Interference Avoidance through Dynamic Downlink OFDMA Subchannel Allocation using Inter-cell Coordination", IEEE 67th Vehicular Technology Conference, (VTC
TABLE 10. NOMENCLATURE

\begin{tabular}{|c|c|}
\hline 3GPP & Third Generation Partnership Project \\
\hline ABS & Almost Blank Subframes \\
\hline AMC & Adaptive Modulation and Coding scheme \\
\hline BER & Bit Error Ratio \\
\hline BS & Base Station \\
\hline $\mathrm{CDF}$ & Cumulative Distribution Function \\
\hline CSG & Closed Subscriber Group \\
\hline CQI & Channel Quality Indicator \\
\hline DL & Downlink \\
\hline Eff. reuse & Effective reuse \\
\hline eICIC & enhanced Inter-Cell Interference Coordination \\
\hline eNB & evolved NodeB \\
\hline FD & Frequency Domain \\
\hline FFR & Fractional Frequency Reuse \\
\hline G-SINR & Geometric SINR \\
\hline GSM & Global System for Mobile communication \\
\hline HetNet & Heterogeneous Network \\
\hline $\mathrm{HI}$ & High Interference \\
\hline HII & High Interference Indicator \\
\hline ICIC & Inter-Cell Interference Coordination \\
\hline ISD & Inter-Site Distance \\
\hline ISI & Inter-Symbol Interference \\
\hline IOI & Interference Overload Indicator \\
\hline LTE & Long-Term Evolution \\
\hline LTE-A & LTE Advanced \\
\hline LP & Linear Programming \\
\hline MCNF & Minimum Cost Network Flow \\
\hline MIMO & Multiple-Input Multiple-Output \\
\hline MINLP & Mixed Integer non-Linear Programming \\
\hline OFDM & Orthogonal Frequency Division Multiplexing \\
\hline OFDMA & Orthogonal Frequency Division Multiple Access \\
\hline PAPR & Peak-to-Average Power Ratio \\
\hline $\mathrm{PF}$ & Proportional Fairness \\
\hline QAM & Quadrature Amplitude Modulation \\
\hline QoS & Quality of Service \\
\hline QSPK & Quadrature Phase Shift Keyed \\
\hline RB & Resource Block \\
\hline $\mathrm{RF}$ & Radio Frequency \\
\hline RNTP & Relative Narrowband Transmitted Power \\
\hline $\mathrm{RR}$ & Round Robin \\
\hline RRM & Radio Resource Management \\
\hline RS & Reference Symbol \\
\hline SC-FDMA & Single-Carrier Frequency Division Multiple Access \\
\hline SD & Spatial Domain \\
\hline SINR & Signal-to-Interference and Noise Ratio \\
\hline SISO & Single-Input Single-Output \\
\hline $\mathrm{TD}$ & Time Domain \\
\hline TTI & Transmission Time Interval \\
\hline UE & User Equipment \\
\hline UL & Uplink \\
\hline WiMAX & Worldwide Interoperability for Microwave Access \\
\hline
\end{tabular}


Spring), vol., no., pp.1630-1635, May 2008.

[32] S. Kyuho, C. Song, G.Veciana, "Dynamic association for load balancing and interference avoidance in multi-cell networks," IEEE Transactions on Wireless Communications, vol.8, no.7, pp.3566-3576, Jul. 2009.

[33] R1-061374, Ericsson, "Downlink inter-cell interference coordination/avoidance - evaluation of frequency reuse", 3GPP TSG-RAN WG1 \#45, Shanghai, China, May 2006.

[34] R1-061375, Ericsson, "Uplink inter-cell interference coordination/avoidance - evaluation of frequency reuse", 3GPP TSG-RAN WG1 \#45, Shanghai, China, May 2006.

[35] G. Fodor, C. Koutsimanis, A. Rácz, N. Reider, A. Simonsson, W. Müller, "Inter-cell Interference Coordination in OFDMA Networks and in the 3GPP Long Term Evolution Systems", Journal of Communications, vol.4, no.7, pp.445-453, Aug. 2009.

[36] A. Atamtürk, G.L. Nemhauser, M.W.P. Savelsbergh, "Conflict graphs in solving integer programming problems", European Journal of Operational Research, pp.40-55, 2000.

[37] M. L. Fisher, "The Lagrangian Relaxation Method for Solving Integer Programming Problems", Management Science, NFORMS, vol.27, no.1, pp.1-18, Jan. 1981.

[38] A. Atamtürk, G. L. Nemhauser, .W.P. Savelsbergh, "The mixed vertex packing problem", Mathematical Programming, Springer-Verlag, vol.89, no.1, pp.35-53, Nov. 2000.

[39] M. Rahman, H. Yanikomeroglu, "Inter-Cell Interference Coordination in OFDMA Networks: A Novel Approach Based on Integer Programming, IEEE 71st Vehicular Technology Conference (VTC-Spring), vol., no., pp.1-5, May 2010.

[40] LP_sovle Optimization Tool (2011). Version 5.5.2.1. [Online]. Available: http://lpsolve.sourceforge.net/.

[41] ILOGCPLEX: High-performance Software for Mathematical Programming and Optimization (2009) Version V12.4 [Online]. Available: http://www.ilog.com/products/cplex/

[42] S. Agmon, "The relaxation method for linear inequalities", Canadian Journal of Mathematics, pp. 382-392, 1954

[43] A. Schrijver, "Theory of Linear and Integer Programming", WileyInterscience Series in Discrete Mathematics Optimization, New York, pp. 266, 1986.

[44] A. B. Sediq, R. Schoenen, H. Yanikomeroglu, G. Senarath, and Z. Chao, "A novel distributed inter-cell interference coordination scheme based on projected subgradient and network flow optimization", IEEE International Symposium on Personal, Indoor and Mobile Radio Communications (PIMRC), vol., no., pp.11-14, Sep. 2011.

[45] A. B. Sediq, R. Schoenen, H. Yanikomeroglu, G. Senarath, “A nearoptimum, dynamic and distributed inter-cell interference coordination scheme with polynomial complexity using projected subgradient and network flow optimization", under review in IEEE Transactions on Vehicular Technology, 2012.

[46] C. Kosta, B. Hunt, A. Quddus, and R. Tafazolli, "A low-complexity distributed Inter-cell Interference Coordination (ICIC) Scheme for the emerging multi-cell HetNets", IEEE 76th Vehicular Technology Conference (VTC 2012-Fall), Sep. 2012.

[47] K. Chawla and X. Qiu, "Quasi-Static resource allocation with interference avoidance for fixed wireless systems", IEEE Journal of Selected Areas of Communication, vol. 17, no. 3, pp. 493-504, Mar. 1999.

[48] M. H. Ahmed, H. Yanikomeroglu, and S. Mahmoud, "Interference management using packet scheduling in broadband wireless access networks", Wiley Journals - Wireless Communications and Mobile Computing, Nov. 2003.

[49] M. Necker, "Local Interference Coordination in Cellular OFDMA Networks", IEEE 66th Vehicular Technology Conference (VTC-Fall), vol., no., pp.1741-1746, Oct 2007.

[50] L. Haipeng, Z. Lei, Z. Xin, Y. Dacheng, "A Novel Multi-Cell OFDMA System Structure using Fractional Frequency Reuse", IEEE 18th Personal International Symposium on Indoor and Mobile Radio Communications, (PIMRC), vol., no., pp.1-5, Sep. 2007.

[51] D. Lucatti, A. Pattavina, V. Trecordi, "Bounds and performance of reuse partitioning in cellular networks", IEEE Computer Societies Conference, vol.1, no., pp.59-66, Mar. 1996.

[52] A. Najjar, N. Hamdi, A. Bouallegue, "Fractional Frequency Reuse Scheme With Two and Three Regions For Multi-cell OFDMA Systems", 17th Telecommunications forum (TELFOR), Serbia, Belgrade, Nov. 2009.

[53] Z. Xie, B. Walke, "Frequency Reuse Techniques for Attaining Both Coverage and High Spectral Efficiency in OFDMA Cellular Systems",
IEEE Wireless Communications and Networking Conference (WCNC), vol., no., pp.1-6, Apr. 2010

[54] L. Dong, Z. Song, L. Wenxin, W. Wenbo, "A frequency reuse partitioning scheme with successive interference cancellation for OFDMA uplink transmission", 20th IEEE International Symposium on Personal, Indoor and Mobile Radio Communication (PIMRC), vol., no., pp.1362-1366, Sep. 2009.

[55] S.W. Halpern, "Reuse partitioning in cellular systems", 33rd IEEE Vehicular Technology Conference, vol.33, no., pp.322- 327, May 1983.

[56] T. T. Nielsen, J. Wigard, P. Mogensen, "On the capacity of a GSM frequency hopping network with intelligent underlay-overlay", IEEE 47th Vehicular Technology Conference (VTC-Spring), vol.3, no., pp.1867-1871 vol.3, May 1997.

[57] K. Begain, G.I. Rózsa, A. Pfening, M. Telek, "Performance Analysis of GSM Networks with Intelligent Underlay-Overlay", IEEE 7th Symposium on Computers and Communications (ISCC), vol., no., pp.135-141, 2002.

[58] M. Sternad, T. Ottosson, A. Ahlen, A. Svensson, "Attaining both coverage and high spectral efficiency with adaptive OFDM downlinks", IEEE 58th Vehicular Technology Conference (VTC-Fall), vol.4, no., pp.2486-2490, Oct. 2003

[59] M. Assaad, "Optimal Fractional Frequency Reuse (FFR) in Multicellular OFDMA System", IEEE 68th Vehicular Technology Conference, (VTC-Fall), vol., no., pp.1-5, Sep. 2008.

[60] N. Himayat, S. Talwar, A. Rao, R. Soni, "Interference management for $4 \mathrm{G}$ cellular standards [WIMAX/LTE UPDATE]", IEEE Communications Magazine, vol.48, no.8, pp.86-92, Aug. 2010.

[61] 3GPP R1-060135, Siemens, "Interference Mitigation by Partial Frequency Reuse", TSG-RAN WG1 Ad Hoc Meeting on LTE, Helsinki, Finland, Jan. 2006.

[62] 3GPP R1-050507, Huawei, "Soft frequency reuse scheme for UTRAN LTE”, TSG RAN WG1 Meeting \#41, Athens, Greece, May 2005.

[63] 3GPP R1-050764, Ericsson, "Inter-cell Interference Handling for EUTRA", TSG-RAN WG1 Meeting \#42, London, UK, Sep. 2005.

[64] M. Rahman, H. Yanikomeroglu, W. Wong, "Interference avoidance with dynamic inter-cell coordination for downlink LTE system", IEEE Wireless Communications and Networking Conference (WCNC), vol., no., pp.1-6, Apr. 2009.

[65] Y. Xiang, J. Luo, E. Schulz, C. Görg, "Performance impact of flexible power arrangement in OFDMA based cellular communication networks", Annals of Telecommunications, vol.63, no.5, pp.271-280, Jun. 2008.

[66] Y. Xiang, J. Luo, "Inter-cell interference mitigation through flexible resource reuse in OFDMA based communication networks," 13th European Wireless Conference (EW), vol., no., pp.1-7, Apr. 2007.

[67] T.D. Novlan, R.K. Ganti, A. Ghosh, J.G. Andrews, , "Analytical Evaluation of Fractional Frequency Reuse for OFDMA Cellular Networks", IEEE Transactions on Wireless Communications, vol.10, no.12, pp.4294-4305, Dec. 2011

[68] R.Y. Chang, Z. Tao, J. Zhang, C.-C.J. Kuo, "A Graph Approach to Dynamic Fractional Frequency Reuse (FFR) in Multi-Cell OFDMA Networks," IEEE International Conference on Communications (ICC), vol., no., pp.1-6, Jun. 2009.

[69] D. Jia, G. Wu, S. Li, G.Y. Li, X. Zhu, "Dynamic Soft-Frequency Reuse with Inter-Cell Coordination in OFDMA Networks", 20th International Conference on Computer Communications and Networks (ICCCN), vol., no., pp.1-6, Aug. 2011.

[70] 3GPP R1-050594, Alcatel, "Multi-cell Simulation Results for Interference Co-ordination in new OFDM DL", TSG RAN WG1 LTE Ad Hoc on LTE, Sophia Antipolis, France, Jun. 2005.

[71] 3GPP R1-050808, ETRI, "Inter-cell interference management in practical environments", TSG RAN WG1 Meeting \#42, London, UK, Sep. 2005

[72] V. Chandrasekhar and J. Andrews, "Femtocell networks: A survey," IEEE Communication Magazine, vol. 46, no. 9, pp. 59-67, Sep. 2008.

[73] L. Taeyoung, Y. Jisun, L. Sangtae, S. Jitae, "Resource allocation analysis in OFDMA femtocells using Fractional Frequency Reuse", IEEE 21st International Symposium on Personal Indoor and Mobile Radio Communications (PIMRC), , vol., no., pp.1224-1229, 26-30 Sep. 2010.

[74] I. Guvenc, M. -R. Jeong, F. Watanabe, H. Inamura, "A hybrid frequency assignment for femtocells and coverage area analysis for co-channel operation", IEEE Communications Letters, vol.12, no.12, pp.880-882, Dec. 2008.

[75] D. Lopez-Perez, A. Ladanyi, A. Juttner, J Zhang; , "OFDMA femtocells: 
A self-organizing approach for frequency assignment", IEEE $20^{\text {th }}$ International Symposium on Personal, Indoor and Mobile Radio Communications(PIMRC), vol., no., pp.2202-2207, Sep. 2009.

[76] C. Kosta, A. Imran, A.U. Quddus, R. Tafazolli, "Flexible Soft Frequency Reuse Schemes for Heterogeneous Networks (Macrocell and Femtocell)", IEEE $73^{\text {rd }}$ Vehicular Technology Conference (VTC Spring), vol., no., pp.1-5, May 2011.

[77] T. Zahir, K. Arshad, A. Nakata, K. Moessner, "Interference Management in Femtocells", IEEE Communications Surveys \& Tutorials, vol. PP, no.99, pp.1-19, 2012.

[78] 3GPP R1-060586, Ericsson, NTT, DoCoMo, "Downlink and uplink inter-cell interference co-ordination/avoidance - impact on the specifications", TSG-RAN WG1 meeting \#44, Denver, USA, Feb. 2006.

[79] R. Ghaffar, R. Knopp, "Fractional frequency reuse and interference suppression for OFDMA networks," 8th International Symposium on Modeling and Optimization in Mobile, Ad Hoc and Wireless Networks (WiOpt), vol., no., pp.273-277, Jun. 2010

[80] 3GPP R1-050833, LG Electronics, "Interference mitigation in evolved UTRA/UTRAN", TSG RAN WG1 Meeting \#42, London, UK, Sep. 2005

[81] X. Xiang, F. Liu, Y. Ji, "Simulation based performance evaluation of ICI mitigation schemes for broadband wireless access networks", 11th communications and networking simulation symposium, vol., no., pp.181-187, Apr. 2008

[82] L. Ahlin, J. Zander, Digital communication - systems and methods, Student litteratur, 1997.

[83] C. G. Gerlach, I. Karla, A. Weber, L. Ewe, H. Bakker, E. Kuehn, A. Rao, "ICIC in DL and UL With Network Distributed and Self-Organized Resource Assignment Algorithms in LTE”, Bell Labs Technical Journal, vol. 15(3), no., pp.43-62, Dec. 2010.

[84] 3GPP R1-050738, Siemens, "Interference mitigation - Considerations and Results on Frequency Reuse", TSG-RAN WG1 Meeting \#42, London, UK, Sep. 2005.

[85] 3GPP R1-072762, Nortel, "Further Discussion on Adaptive Fractional Frequency Reuse", 3GPP TSG-RAN WG1 \#49bis, Orlando, FL USA, Jun. 2007.

[86] 3GPP R1-051059, Texas Instruments, Inter-Cell Interference Mitigation for EUTRA, 3GPP TSG RAN WG1, San Diego, USA, Oct. 2005.

[87] F. Xiangning, C. Si, Z. Xiaodong, "An Inter-Cell Interference Coordination Technique Based on Users' Ratio and Multi-Level Frequency Allocations", International Conference on Wireless Communications, Networking and Mobile Computing Conference (WiCom), vol., no., pp.799-802, Sep. 2007.

[88] M. Garey, D. S. Johnson, "Computers and Intractability: A Guide to the Theory of NP-Completeness". W.H. Freeman and Company, 1979.

[89] R. Rajkumar, C. Lee, J. Lehoczky, D. Siewiorek, "Practical solutions for QoS-based resource allocation problems", IEEE 19th Real-Time Systems Symposium, vol., no., pp.296-306, Dec 1998.

[90] Z. Liang, Y. H. Chew,C. C. Ko, "A Linear Programming Solution to Subcarrier, Bit and Power Allocation for Multicell OFDMA Systems", Wireless Communications and Networking Conference (WCNC), vol., no., pp.1273-1278, Apr. 2008.

[91] D. López-Pérez, A. Jüttner, J. Zhang, "Dynamic Frequency Planning Versus Frequency Reuse Schemes in OFDMA Networks", IEEE 69th Vehicular Technology Conference (VTC Spring), vol., no., pp.1-5, Apr. 2009.

[92] M. Rahman, H. Yanikomeroglu, "Multicell Downlink OFDM Subchannel Allocations using Dynamic Intercell Coordination", IEEE 50th Global Telecommunications Conference (GLOBECOM), vol., no., pp.5220-5225, Nov. 2007.

[93] M. Rahman, H. Yanikomeroglu, "Enhancing cell-edge performance: a downlink dynamic interference avoidance scheme with inter-cell coordination", IEEE Transactions on Wireless Communications, vol.9, no.4, pp.1414-1425, Apr. 2010.

[94] G. Li, H. Liu, "Downlink Radio Resource Allocation for Multi-Cell OFDMA System", IEEE Transactions on Wireless Communications, vol.5, no.12, pp.3451-3459, Dec. 2006.

[95] F. Meshkati, H.V. Poor, S.C. Schwartz, "Energy-Efficient Resource Allocation in Wireless Networks", IEEE Signal Processing Magazine, vol.24, no.3, pp.58-68, May 2007.

[96] J. Ellenbeck, C. Hartmann, L. Berlemann, "Decentralized inter-cell interference coordination by autonomous spectral reuse decisions", European Wireless Conference (EW), vol., no., pp.1-7, Jun. 2008.

[97] J. Ellenbeck, H. A. Shatri, C. Hartmann, "Performance of Decentralized Interference Coordination in the LTE Uplink", IEEE 70th Vehicular
Technology Conference (VTC-Fall), vol., no., pp.1-5, Sep. 2009.

[98] A. Ozyagci, O. Ileri, J. Zander, "Distributed multi-channel random access networks with selfish users", 5th International Conference on Cognitive Radio Oriented Wireless Networks \& Communications (CROWNCOM), vol., no., pp.1-5, Jun. 2010.

[99] A. Y. Al-Zahrani, F.R. Yu, "A Game Theory Approach for Inter-Cell Interference Management in OFDM Networks", 2011 IEEE International Conference on Communications (ICC), vol., no., pp.1-5, Jun. 2011.

[100]A. L. Stolyar, H. Viswanathan, "Self-Organizing Dynamic Fractional Frequency Reuse in OFDMA Systems", IEEE 27th Conference on Computer Communications (INFOCOM), vol., no., pp.691-699, Apr. 2008.

[101]A.L. Stolyar, H. Viswanathan, "Self-Organizing Dynamic Fractional Frequency Reuse for Best-Effort Traffic through Distributed Inter-Cell Coordination", IEEE 28th Conference on Computer Communications (INFOCOM), , vol., no., pp.1287-1295, Apr. 2009.

[102]B. Rengarajan, A.L. Stolyar, H. Viswanathan, "Self-organizing Dynamic Fractional Frequency Reuse on the uplink of OFDMA systems," 44th Annual Conference on Information Sciences and Systems (CISS), vol., no., pp.1-6, Mar. 2010.

[103]F. Dominique1, C. G. Gerlach, N. Gopalakrishnan, A. Rao, J.P. Seymour, et al., "Self-Organizing Interference Management for LTE" Bell Labs Technical Journal, vol.15(3), pp.19-42, Dec. 2010.

[104]O. Aliu, A. Imran, M. Imran, B. Evans, "A Survey of Self Organization in Future Cellular Networks", IEEE Communications Surveys \& Tutorials, vol.PP, no.99, pp.1-26, 2012.

[105]3GPP TR 36.423, "X2 Application Protocol (X2AP) (Release 8)", v8.2.0, http://www.3gpp.org, Jun. 2008.

[106]V. Pauli, J. D. Naranjo, E. Seidel, "Heterogeneous LTE Networks and Inter-Cell Interference Coordination", White Paper - Nomor Research $\mathrm{GmbH}$, Munich Germany, Dec. 2010.

[107]3GPP R1-101369, LG Electronics, "Considerations on interference coordination in heterogeneous networks", TSG-RAN WG1 Meeting \#60, San Francisco, USA, Feb. 2010.

[108]H. Holma, A. Toskala, "LTE for UMTS OFDMA and SC-FDMA Based Radio Access", John Wiley \& Sons Ltd, pp.204, 2009.

[109]T.-T. Tran, Y. Shin, O.-S. Shin, "Overview of enabling technologies for 3GPP LTE-advanced", EURASIP Journal of Wireless Communication and Networking, vol. 2012, Feb 2012.

[110]Z. Bharucha, A. Saul, G. Auer, and H. Haas, "Dynamic Resource Partitioning for Downlink Femto-to-Macro-Cell Interference Avoidance", EURASIP Journal of Wireless Communication and Networking, vol. 2010, Article ID 143413, 2010.

[111]V. Chandrasekhar, M. Kountouris, J.G. Andrews, "Coverage in multiantenna two-tier networks", IEEE Transactions on Wireless Communications, vol.8, no.10, pp.5314-5327, Oct 2009.

[112]X. Chu, Y. Wu, D. Lopez-Perez, Xiaofeng Tao , "On Providing Downlink Services in Collocated Spectrum-Sharing Macro and Femto Networks", IEEE Transactions on Wireless Communications, vol.10, no.12, pp.4306-4315, Dec 2011.

[113]3GPP R1-104968, CMCC(Rapporteur), "Summary of the description of candidate eICIC solutions", TSG-WG1 Meeting \#62, Madrid, Spain, Aug 2010

[114]V. Pauli, J. D. Naranjo, E. Seidel, "Inter-Cell Interference Coordination for LTE-A", White Paper - Nomor Research GmbH, Munich Germany, Sep. 2011

[115]R. Madan, J. Borran, A. Sampath, N. Bhushan,; A. Khandekar, J. Tingfang, "Cell Association and Interference Coordination in Heterogeneous LTE-A Cellular Networks", IEEE Journal on Selected Areas in Communications, vol.28, no.9, pp.1479-1489, Dec 2010.

[116]3GPP R1-103264, NTT DOCOMO, "Performance of eICIC with control Channel Coverage Limitation", TSG-RAN WG1 Meeting \#61, Montreal, Canada, May 2010.

[117]D. Lopez-Perez, A. Valcarce, G. de la Roche, Jie Zhang, "OFDMA femtocells: A roadmap on interference avoidance", IEEE Communications Magazine, vol.47, no.9, pp.41-48, Sep. 2009.

[118]M. Morelli, C.-C.J. Kuo, M.-O. Pun, "Synchronization Techniques for Orthogonal Frequency Division Multiple Access (OFDMA): A Tutorial Review", Proceedings of the IEEE, vol.95, no.7, pp.1394-1427, Jul. 2007

[119]A. Ghosh, R. Ratasuk, B. Mondal, N. Mangalvedhe, T. Thomas, "LTEadvanced: next-generation wireless broadband technology [Invited Paper]", IEEE Wireless Communications, , vol.17, no.3, pp.10-22, Jun. 2010. 
[120]D. P. Palomar, C. Mung, "A tutorial on decomposition methods for network utility maximization", IEEE Journal on Selected Areas in Communications, vol.24, no.8, pp.1439-1451, Aug. 2006.

[121]Z. Hasan, H. Boostanimehr, V. K. Bhargava, "Green Cellular Networks: A Survey, Some Research Issues and Challenges", IEEE Communications Surveys \& Tutorials, , vol.13, no.4, pp.524-540, Fourth Quarter 2011.

[122] G.Y. Li, X. Zhikun, X. Cong, Y. Chenyang, Z. Shunqing, C. Yan, et al, "Energy-efficient wireless communications: tutorial, survey, and open issues", IEEE Wireless Communications, vol.18, no.6, pp.28-35, Dec 2011.

[123]S. Cui, A. Goldsmith, A. Bahai, "Energy-constrained modulation optimization", IEEE Transactions on Wireless Communications, vol. 4, no. 5, pp. 2349-2360, Sep. 2005.

[124]J.C. Ikuno, M. Wrulich, M. Rupp, "System Level Simulation of LTE Networks", IEEE 71st Vehicular Technology Conference (VTC-Spring), vol., no., pp.1-5, May 2010.

[125]M. Necker, "Towards Frequency Reuse 1 Cellular FDM/TDM Systems," 9th ACM/IEEE international symposium on Modeling analysis and simulation of wireless and mobile systems (MSWiM), pp.338-346, Oct. 2006.

[126]T. Girici, C. Zhu, J. Agre and A. Ephremides, "Proportional fair scheduling algorithm in OFDMA-based wireless systems with QoS constraints", IEEE Journal of Networks Communications, vol.12, no.1, pp.30-42, Feb. 2010. 